\title{
The Carolina Bays of Ridge Spring, SC
}

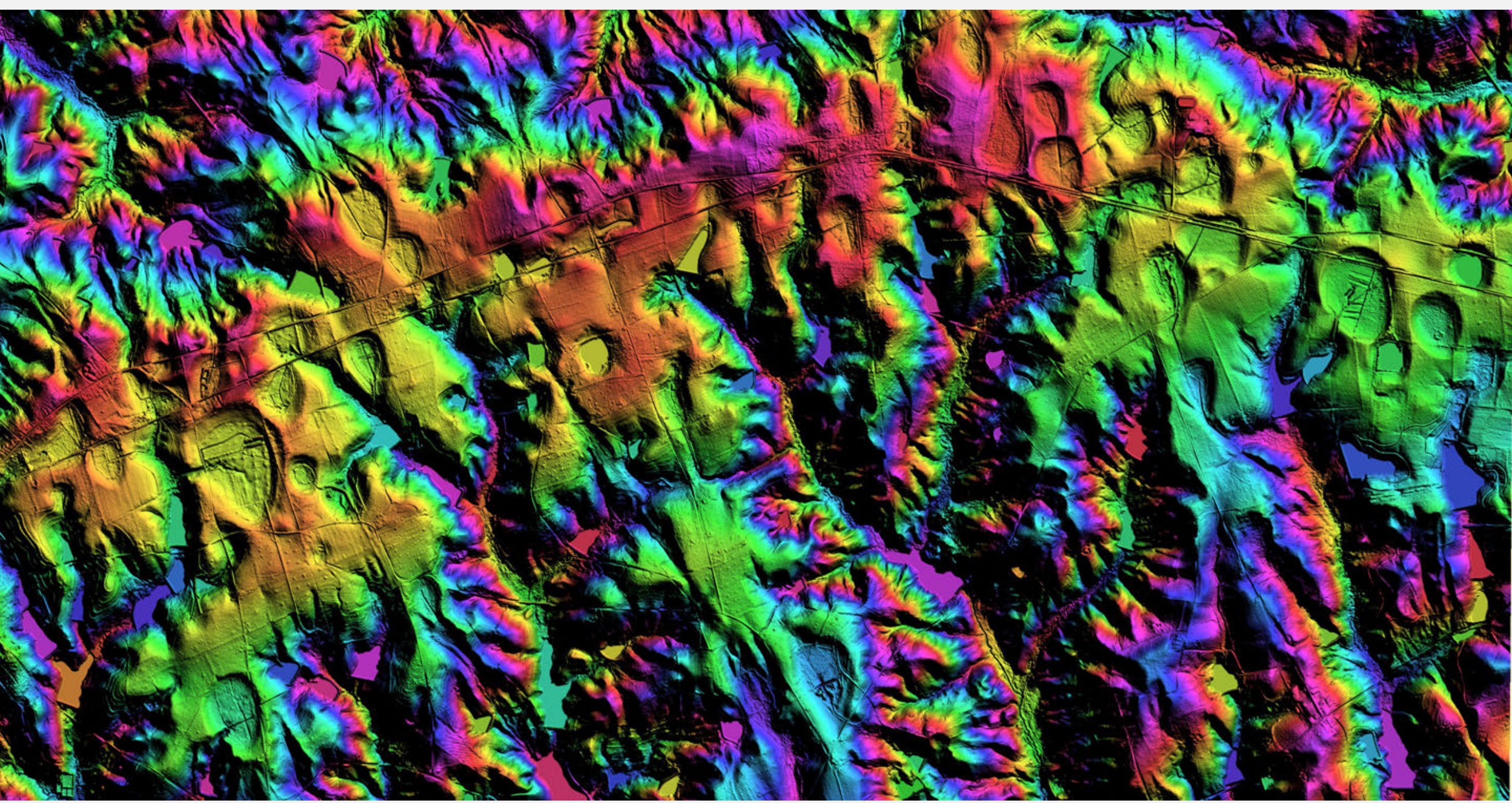

Paper No. 19-10

Michael E. Davias www.cintos.org
2017 GSA Southeastern Section Meeting Richmond, VA 30-31 March, 2017 


\section{THE CAROLINA BAYS OF RIDGE SPRING, SC}

A narrow, sinuous terrace known locally as The Ridge forms a contiguous drainage divide arcing 100 $\mathrm{km}$ from Augusta, GA, northeastward toward Columbia, SC. A new one-meter-resolution digital elevation map was crafted for the terrace using LiDAR data from the SC Department of Natural Resources, providing a crisp perspective of the surficial features present. From their lofty perch at $\sim 200$ $\mathrm{m}$ elevation, the low-relief terrain surrounding Ridge Spring, SC represent a surviving island of flat Cretaceous terrace that is being encroached upon by headward fluvial erosion. Edisto River basin headwaters are eating away the southeastern flank, while along the northwestern flank, tributaries of the upper Santee River drainage basin are collaborating with those of the Savanah River to remove the divide. Of the 45,000+ East Coast bays in the Carolina Bay Geospatial Survey, only 171 bays exist at elevations above $185 \mathrm{~m}$. The Ridge Spring terrace is home to 160 of those high-elevation bays, making the assemblage unique in many ways. These bays maintain robust conformance to the archetype "baySouth" teardrop planform common to over 16,000 neighboring bays; their major axis range from $1.22 \mathrm{~km}$ down to $140 \mathrm{~m}$, with a mean of $380 \mathrm{~m}$; the orientations of that axis range from 148ㅇ to 165, with a mean of 154․ The bays of Ridge Spring are visualized in LiDAR as basins recessed into a surrounding pediment (here, the terrace), exhibit no raised southeastern rim, and have no aeolian dune formations in their vicinity. The headward erosion has been dissecting the terrace since the time of bay formation, as the LiDAR elucidates a history of systematic bay destruction. Some clearly-defined bays have been penetrated by headward erosion, and are no longer hydraulically closed. Former closed-rim bays - recognizable by surviving rim fragments - have become mere "headwater basins". At some point in the future the last vestiges of the terrace surface and the imbedded Carolina bays will be gone. How long will that take? These observations indicate that Carolina bays are not wispy, ephemeral shorelines, but rather represent the surficial expression of robust structures deeply rooted into the landscape. Ridge Spring represents an ideal locale to investigate the burial chronology of Cretaceous strata by surficial sands using Beryllium-10 cosmogenic exposure techniques. 


\section{Goals of Talk}

- Ridge Spring, SC Cretaceous Terrace Remnant

- 200 bays

- Valley Head Basins - Juvenile Carolina bays?

- Geomorphology hypothesis

- Future directions

- All work product freely available @ cintos.org 


\section{"No one has yet invented an explanation which will fully account for all the facts observed" Douglas Johnson}

"Their very randomness of grouping and scatter demands an explanation. As a statistical phenomenon, they deserve to be studied statistically." W.C. Rasmussen

\section{A comprehensive survey might provide the statistics to inspire that "invention".}




\section{Carolina Bay Survey}

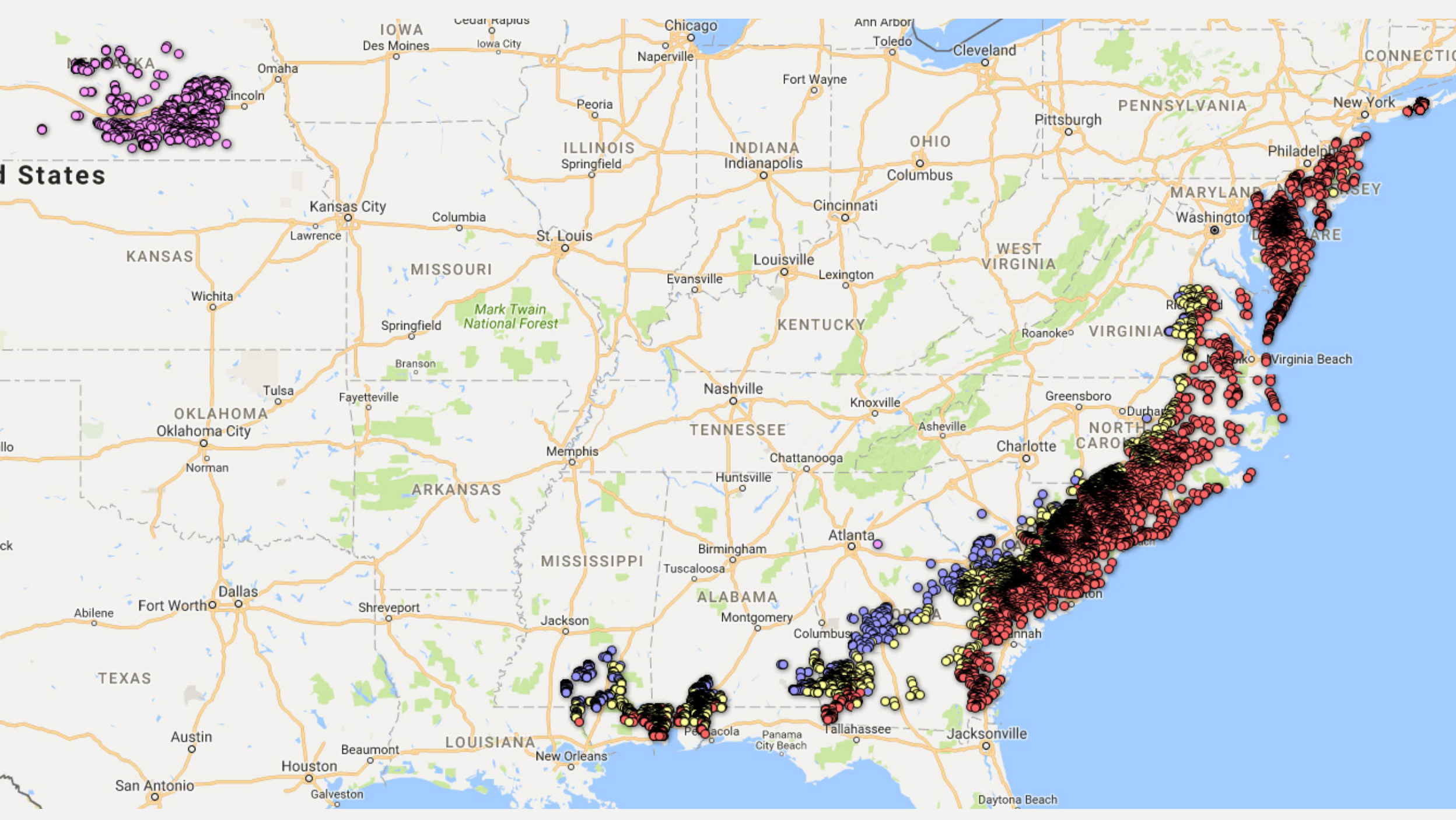

http: //cintos .org/SurveyBayMap 


\section{Carolina Bay Survey}

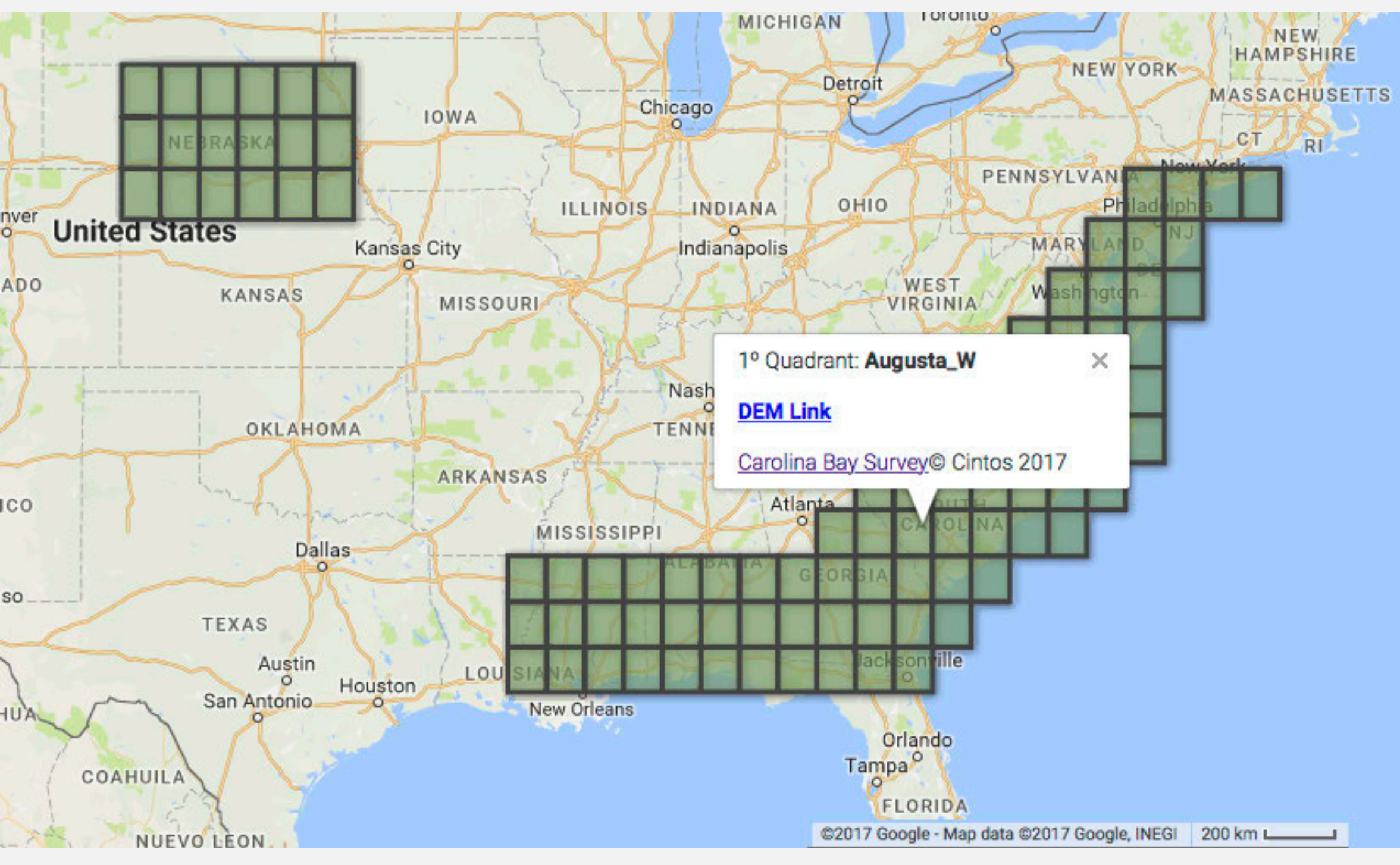

http://cintos.org/SurveyQuadMap 


\section{USGS Augusta_W 10 Quad - 10m DEM}

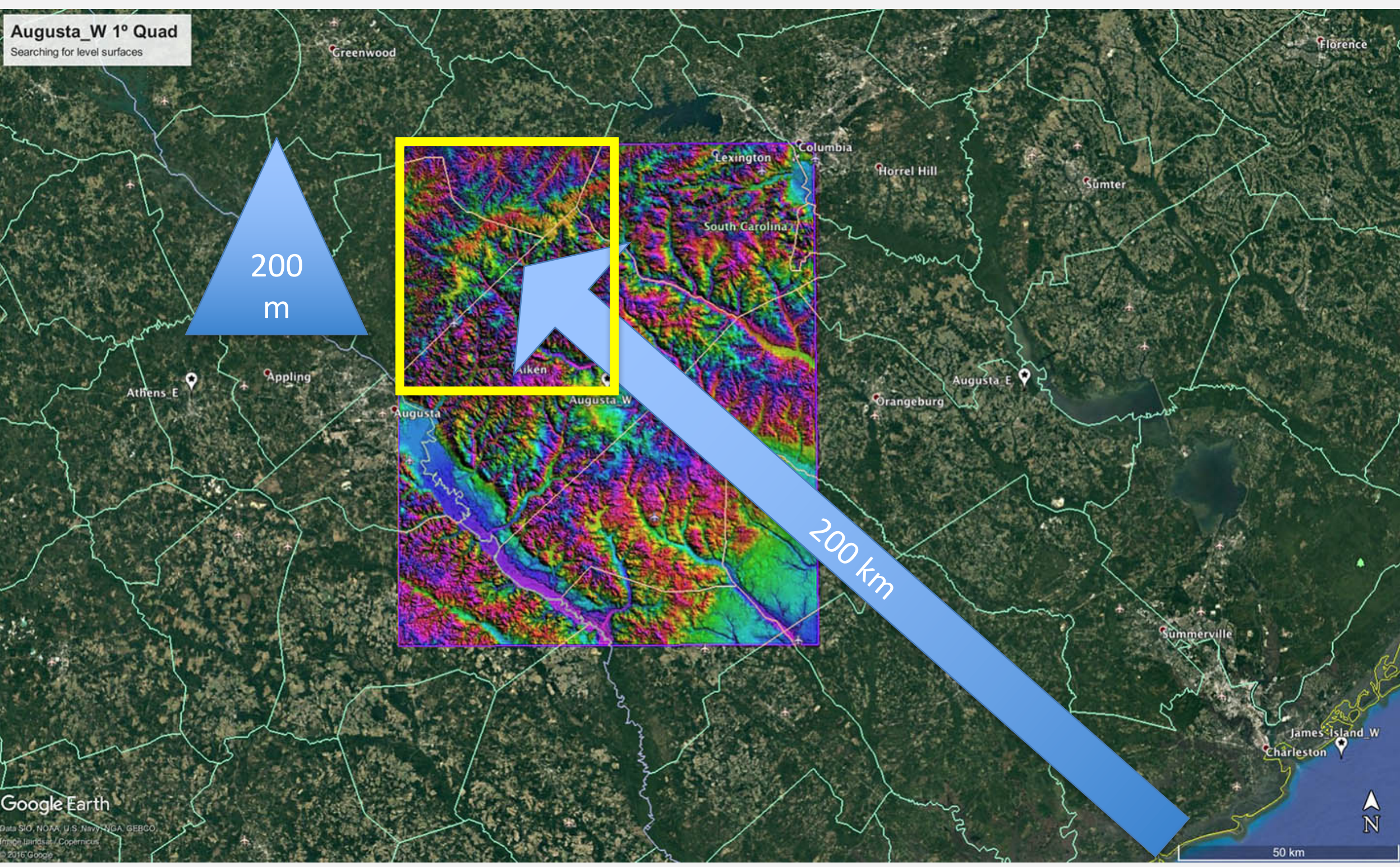

Search for "flat" remnant terrace surfaces which may hold bays 


\section{"The Ridge"}

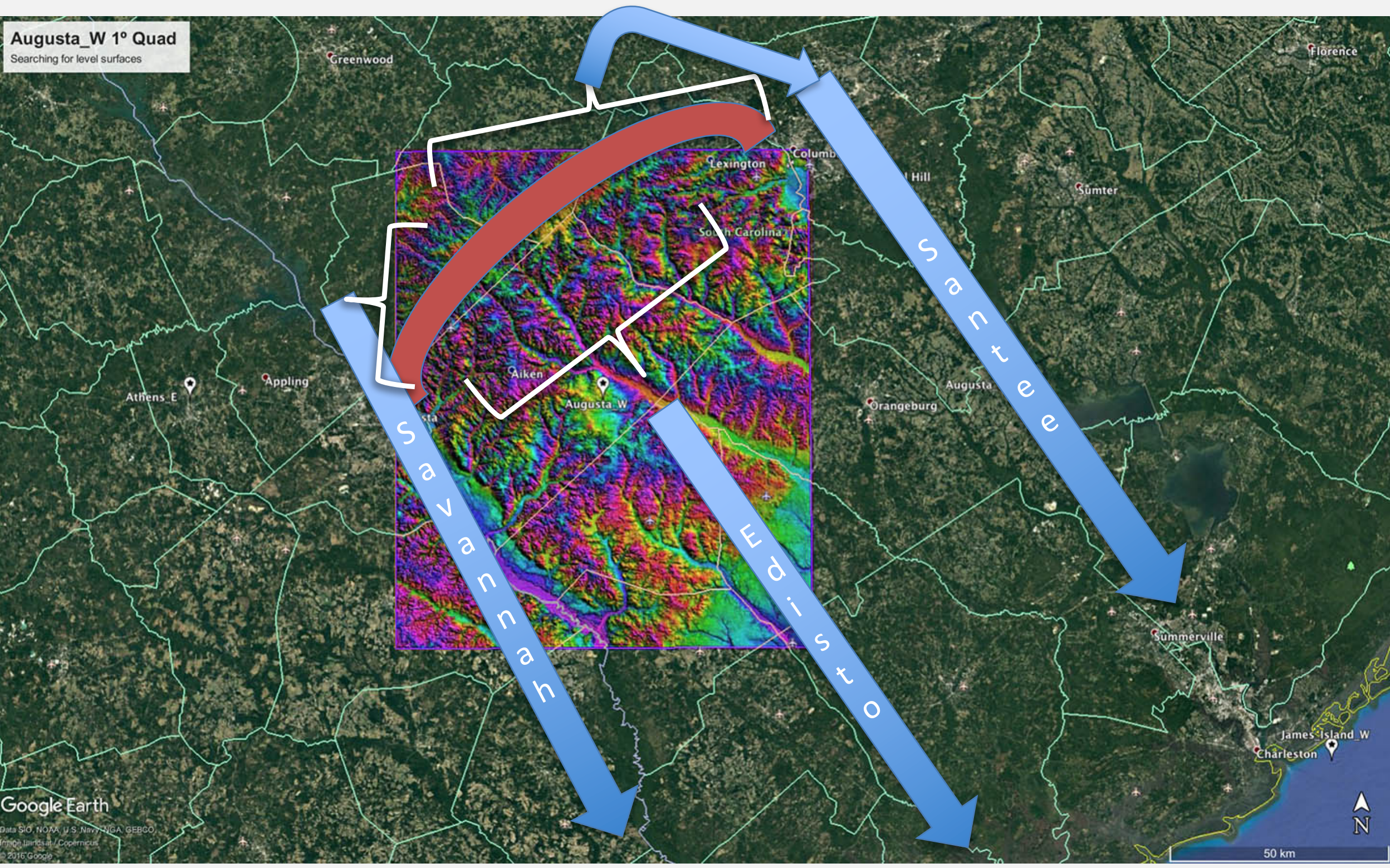

$115 \mathrm{~km}$ of continuous drainage divide between Augusta and Columbia 


\section{Ridge Spring Elevation Profile}

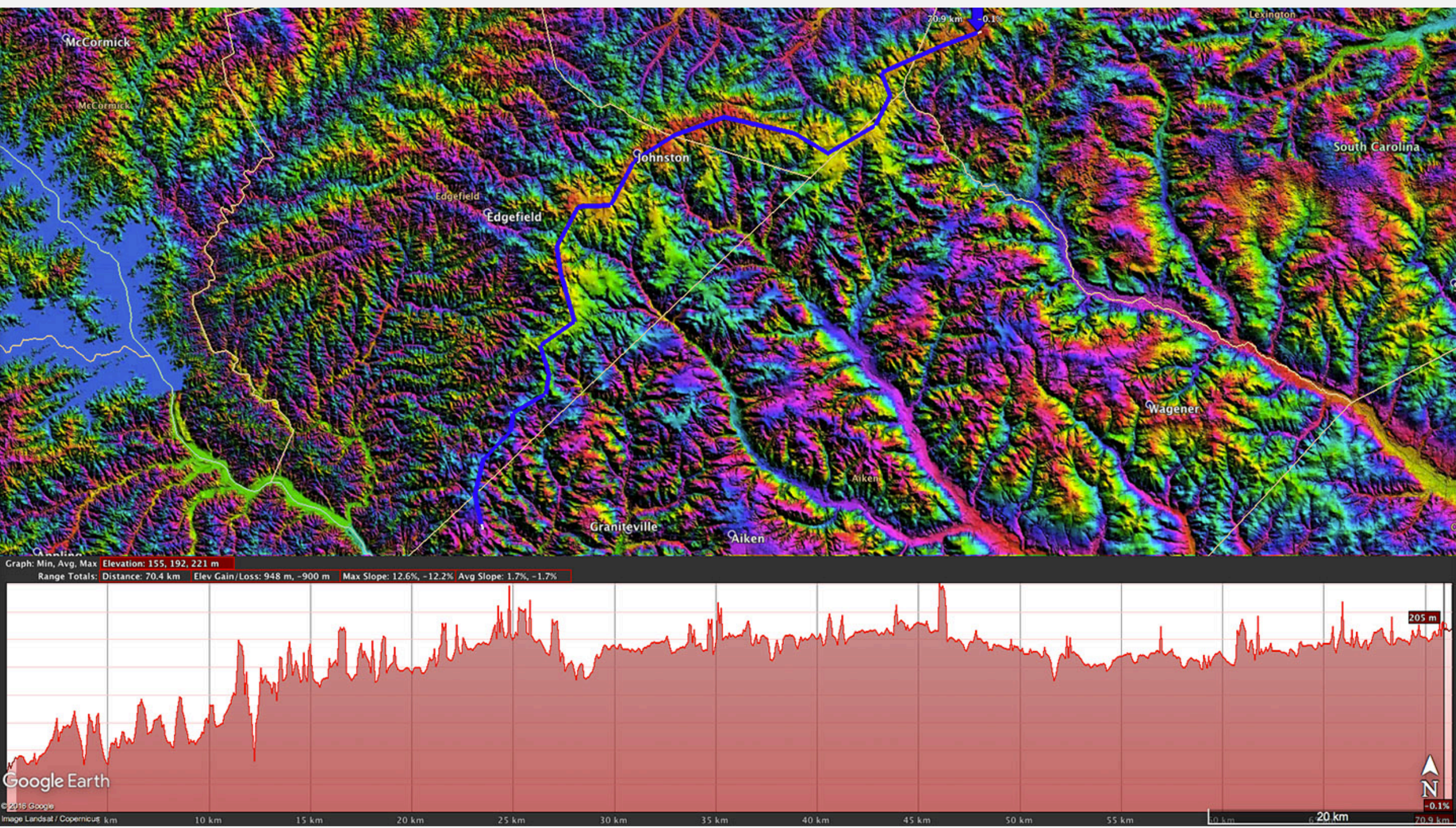

Distance: 70.4 km

Elevation: $155 \mathrm{~m}$ min,

221 m max, 192 m avg 


\section{Ridge Spring Cretaceous Terrace}

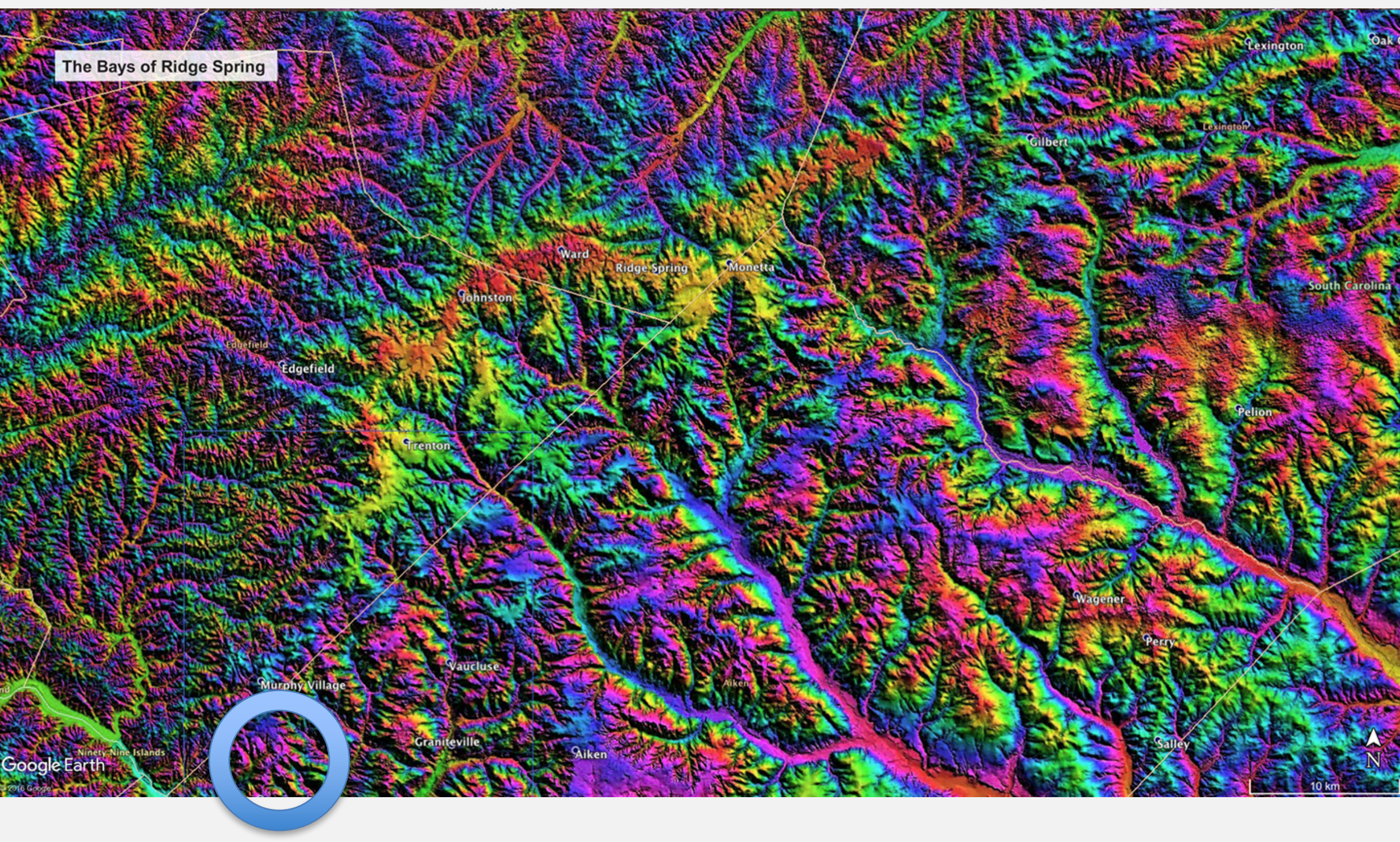

Traversing The Ridge from Augusta to Columbia 


\section{Mathis Lake, Northeast Augusta Suburbs}

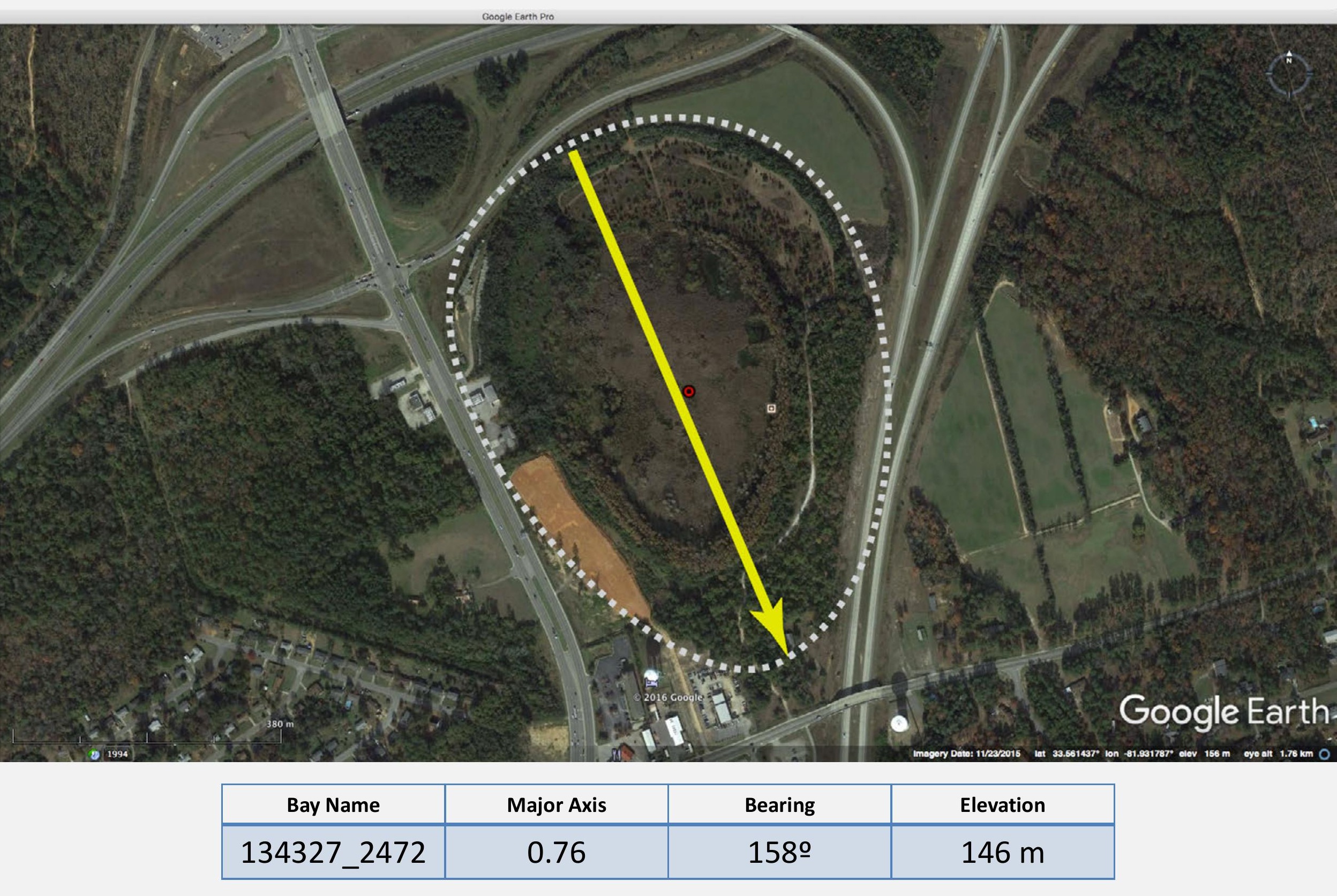




\section{Mathis Lake Bay, Augusta Suburbs}

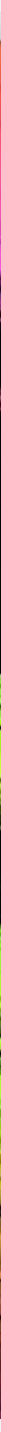

Tiled DEM using Hue-saturation-value (hsv) rendering, 20x elevation exaggeration. USGS sub-m resolution data rendered at 1.5 or $3 \mathrm{~m}$ for network presentation through GE. 


\section{baySouth Archetype Template @ 0o}

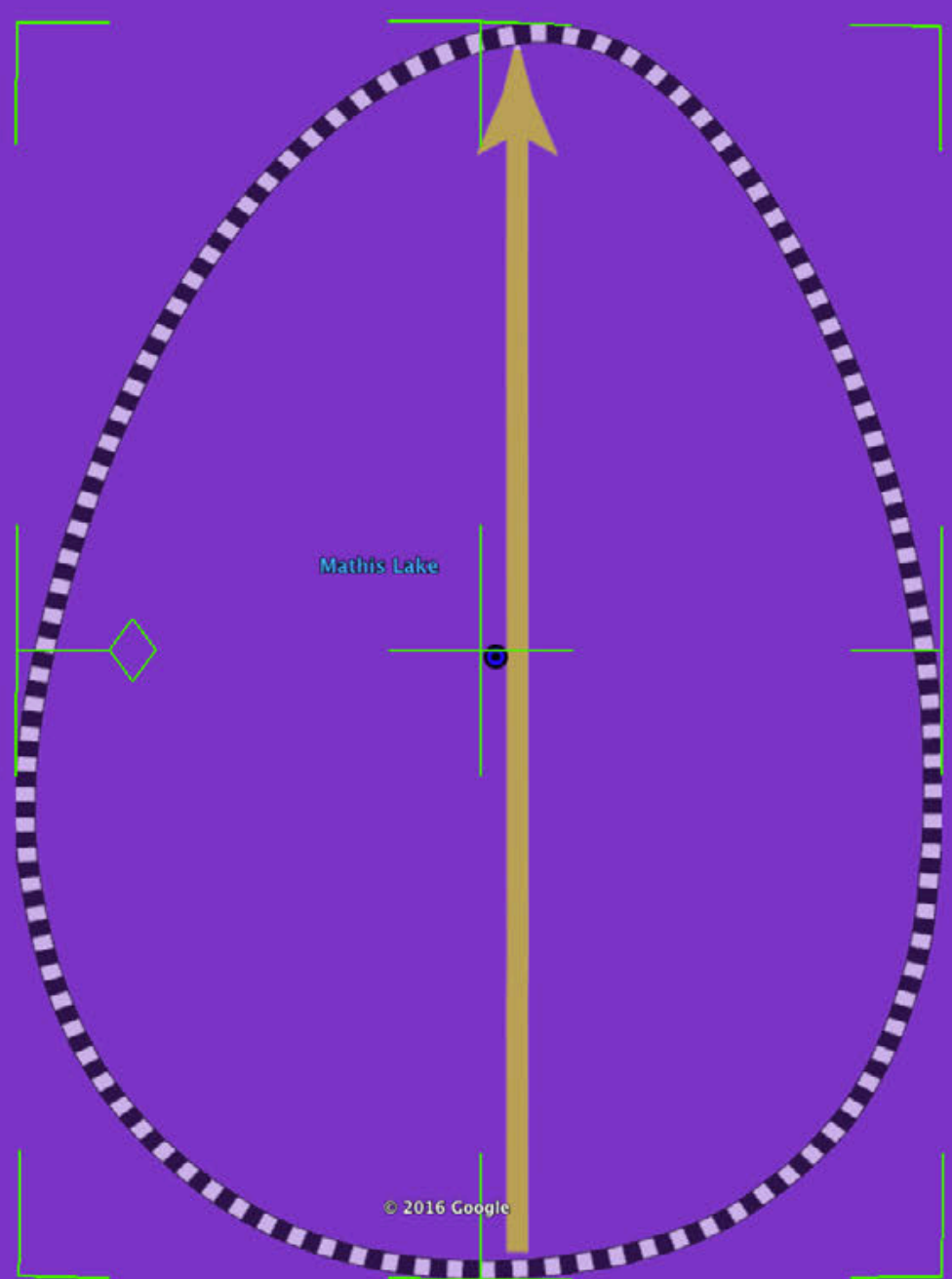

Google Earth

Please reference movie to view GroundOverlay manipulation:

https://gsa.confex.com/gsa/2017SE/webprogram/Handout/Paper291016/MathisBayMeasurment.pdf 


\section{Keyhole Markup Language Data in GroundOverlay}

<GroundOverlay>

<name>134327 2472</name>

$<$ Icon $>$

$<$ href $\nmid$ http: //cintos.org/ge/overlays/baysouth.png $</$ href $>$

$</$ Icon $>$

$<$ LatLonBox $>$

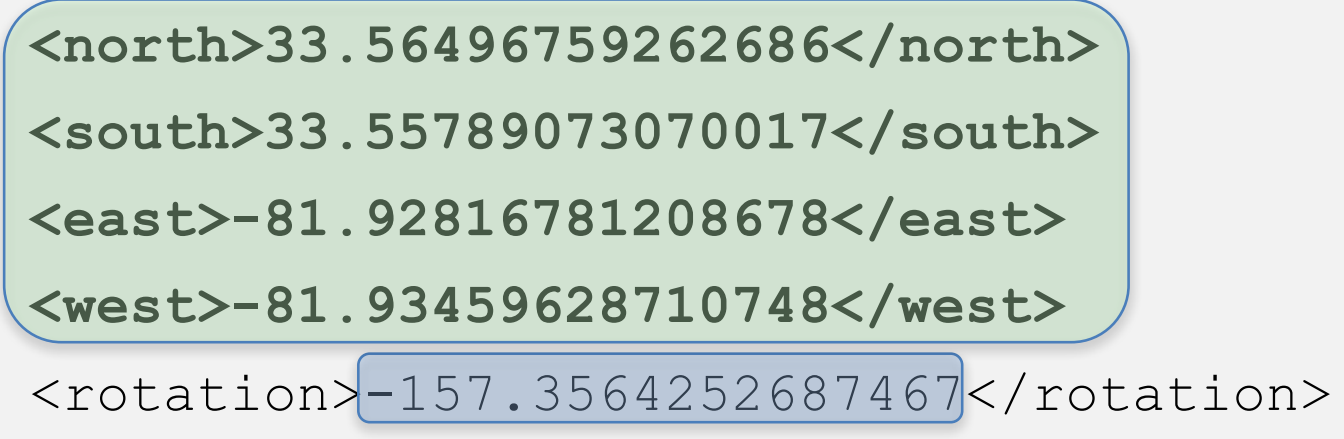

$</$ GroundOverlay $>$

The GroundOverlay meta data's bounding box defines (with a bit of trig) the major and minor axis of the bay, a bay center, and an approximate surface area. Coordinates define the box with zero rotation applied. 


\section{GroundOverlay LatLonBox Computations}

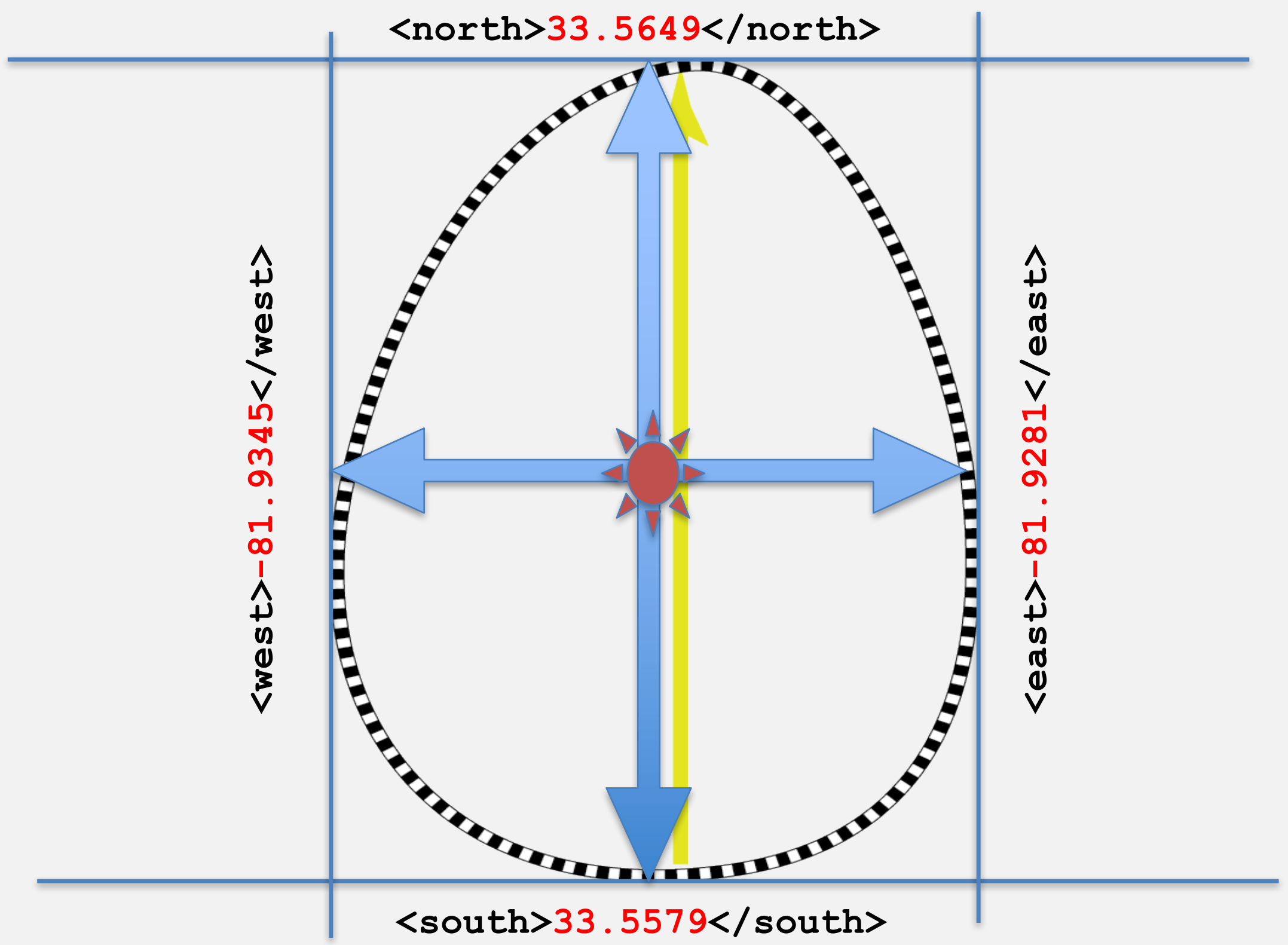


GroundOverlay LatLonBox Computations

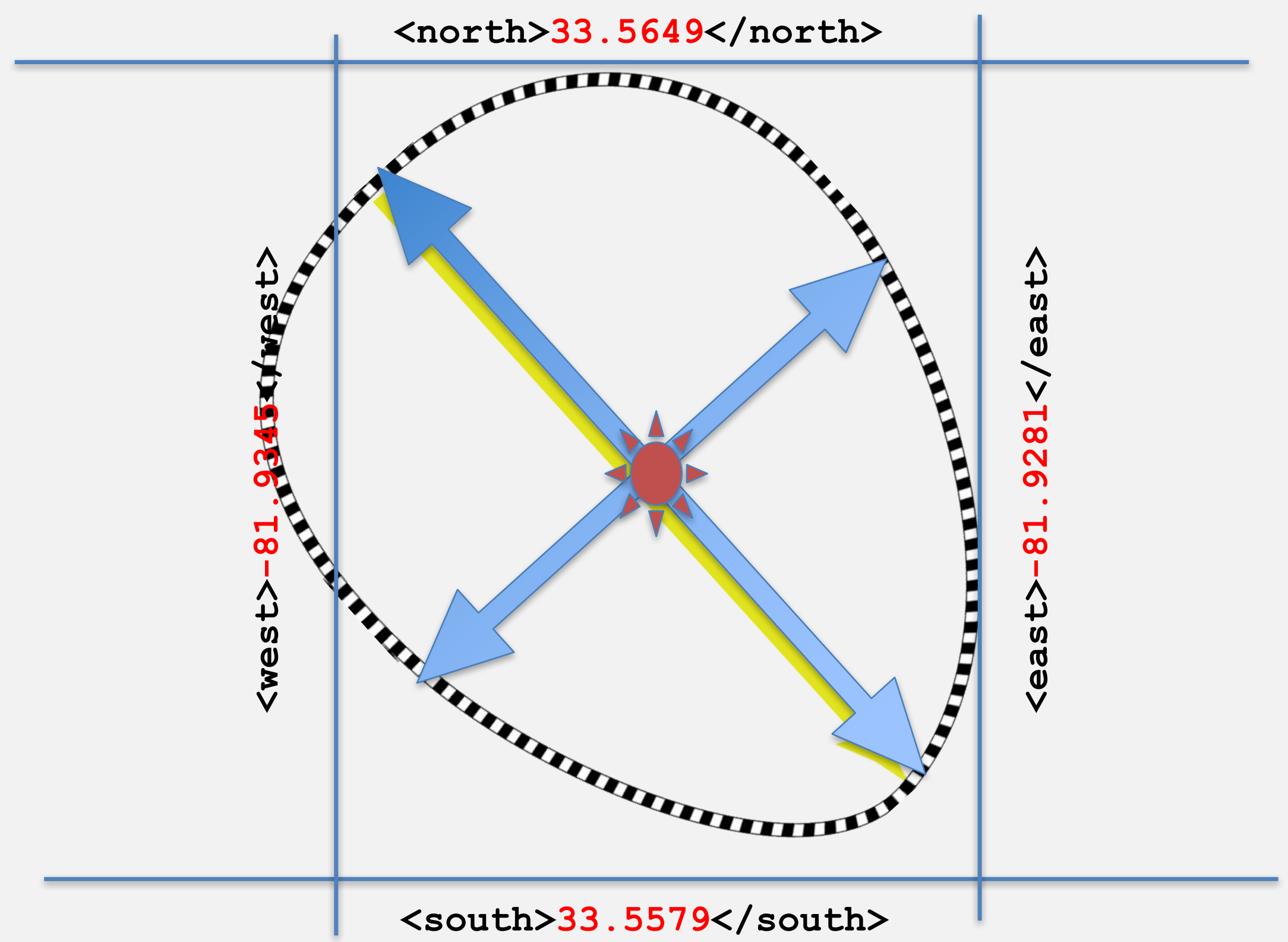




\section{Loading Fusion Table: Carolina Bay Geospatial Survey}

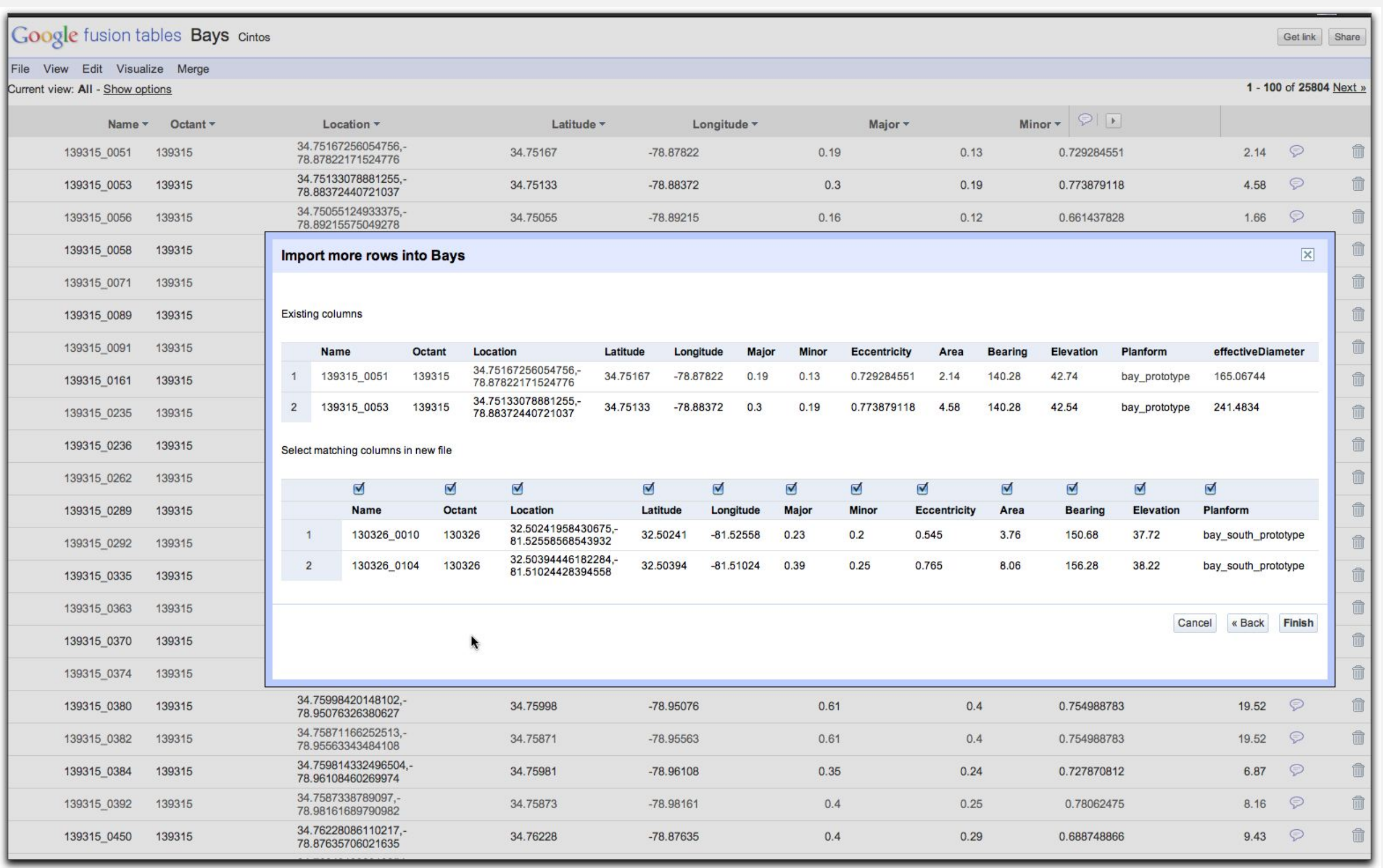

An exclusive index is generated for the bay based on lat \& lon, and the summary geospatial data is uploaded into a Google FusionTable. The table today has just under 44,000 entries. 


\section{Aiken, SC}

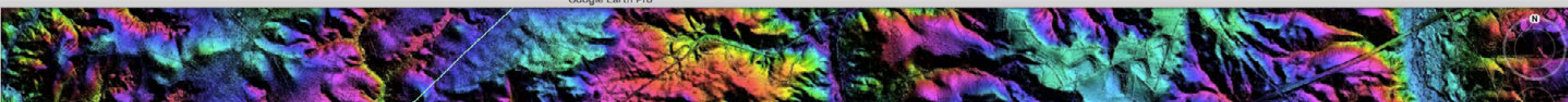

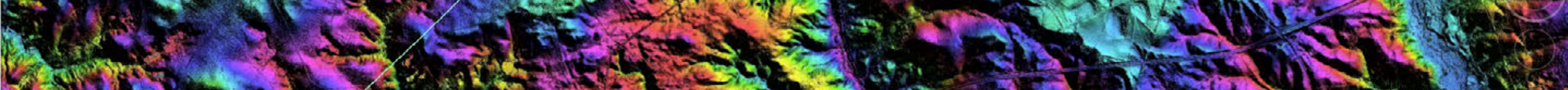
1.1.

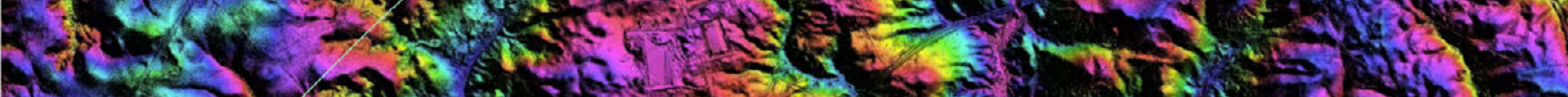

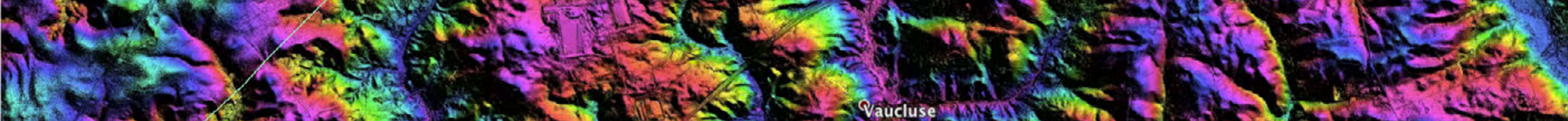

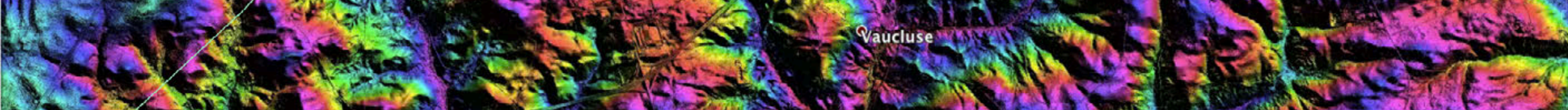

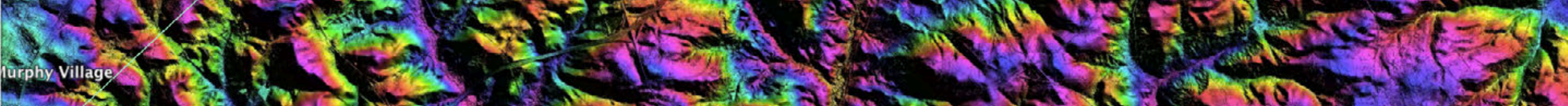

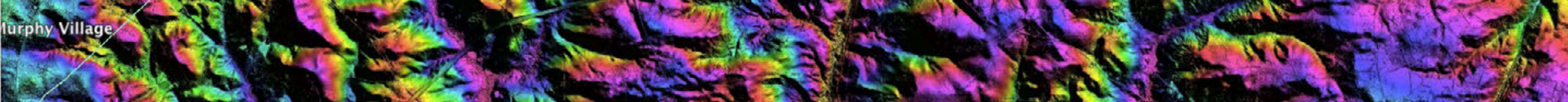

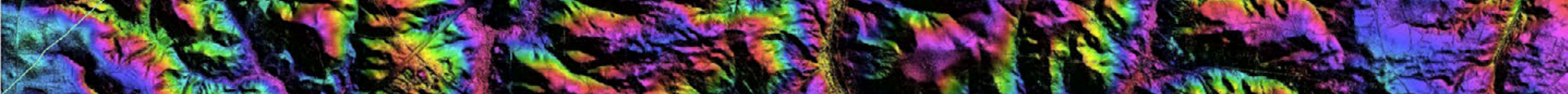

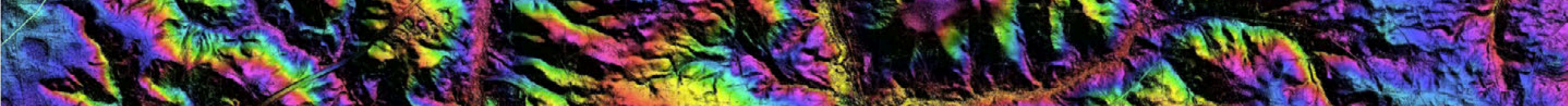

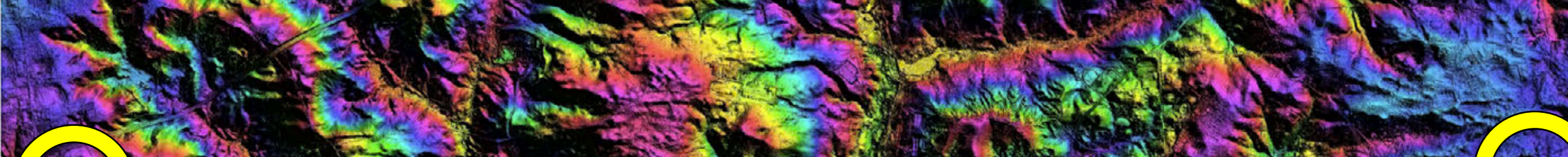

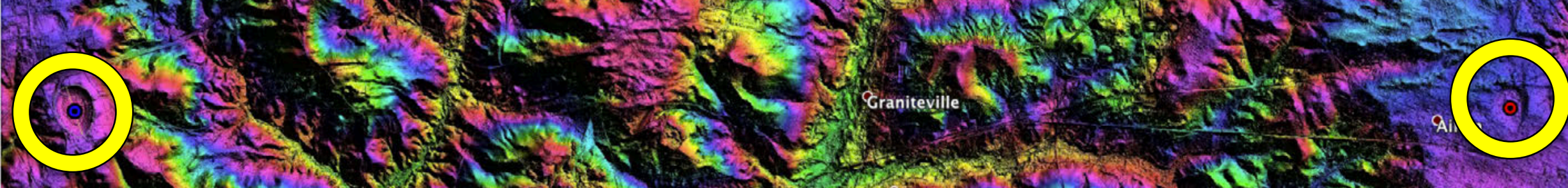

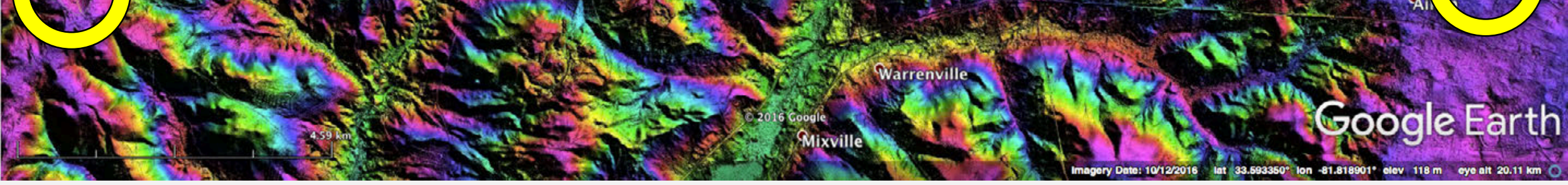

Bay in Aiken, $20 \mathrm{~km}$ due east, at same elevation as Mathis Bay 


\section{Aiken, SC}

Google Earth Pro

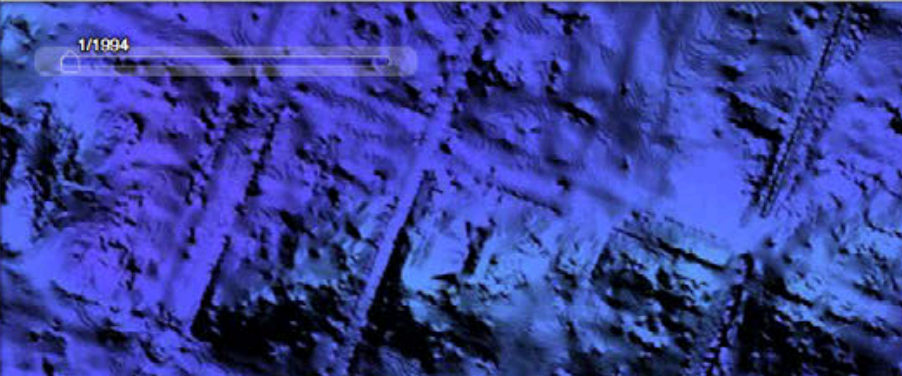

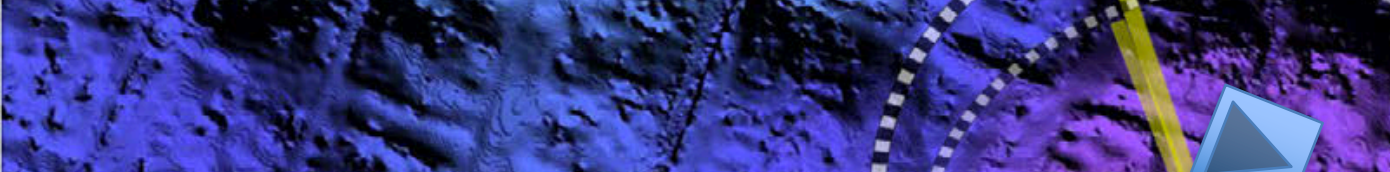

$+x^{2}+x^{2}+3=0$

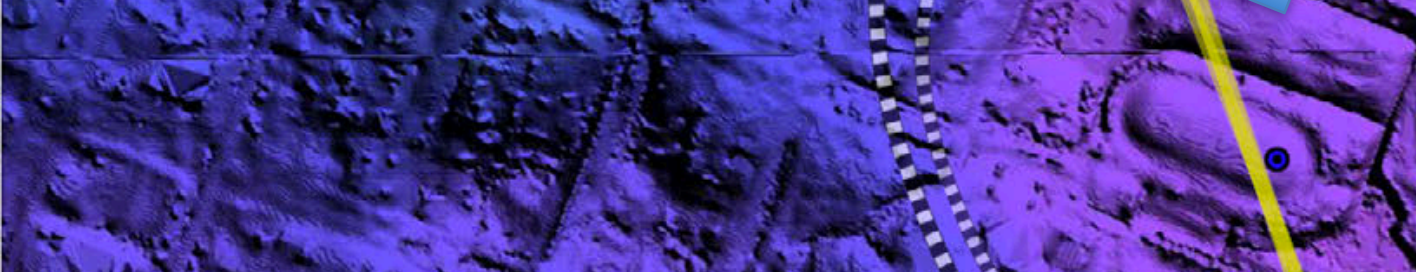

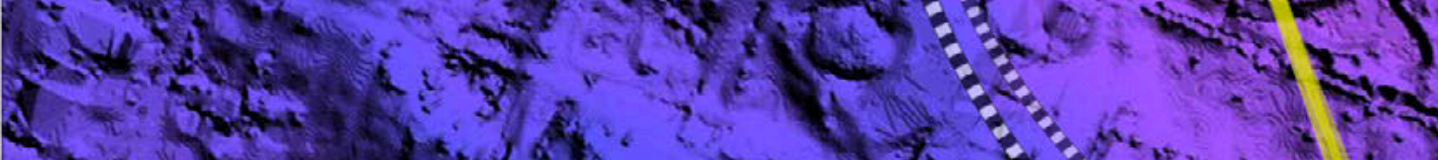

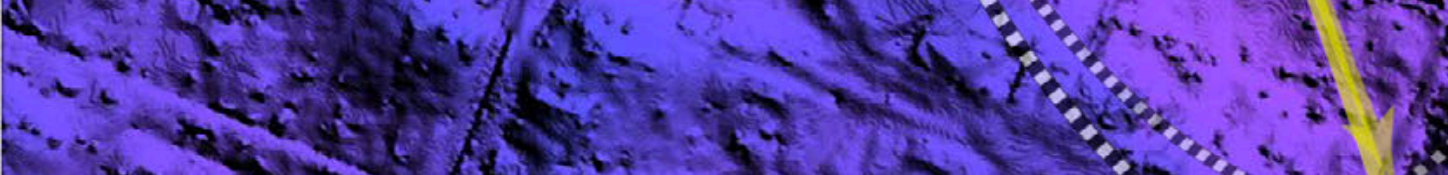

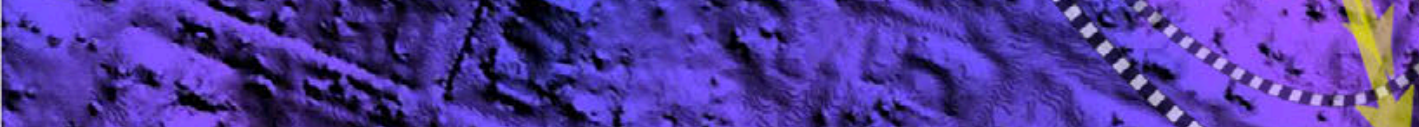

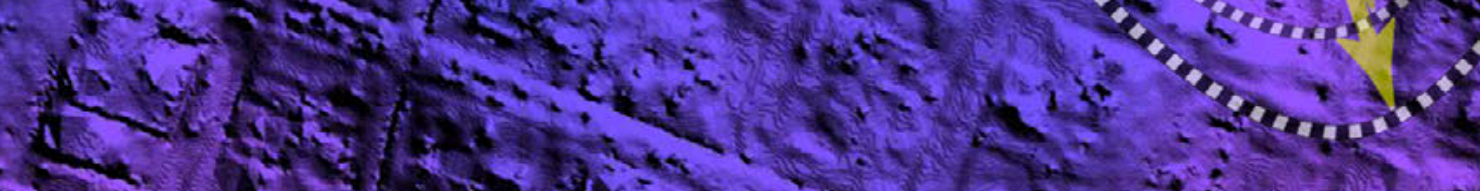

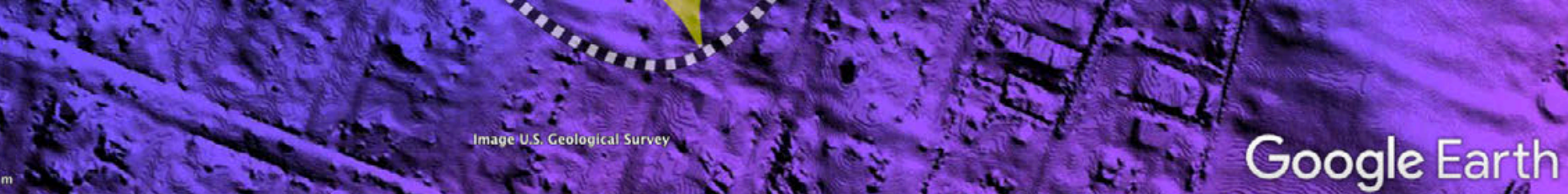

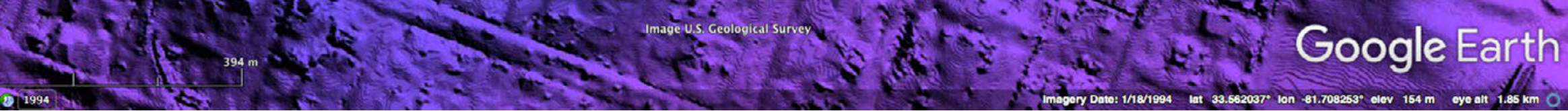

\begin{tabular}{|c|c|c|c|}
\hline Bay Name & Major Axis & Bearing & Elevation \\
\hline 134326_2483 & 0.61 & 159 o & $155 \mathrm{~m}$ \\
\hline
\end{tabular}




\section{Aiken, SC}

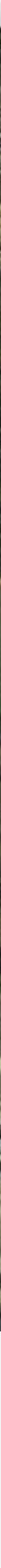




\section{Aiken, SC}

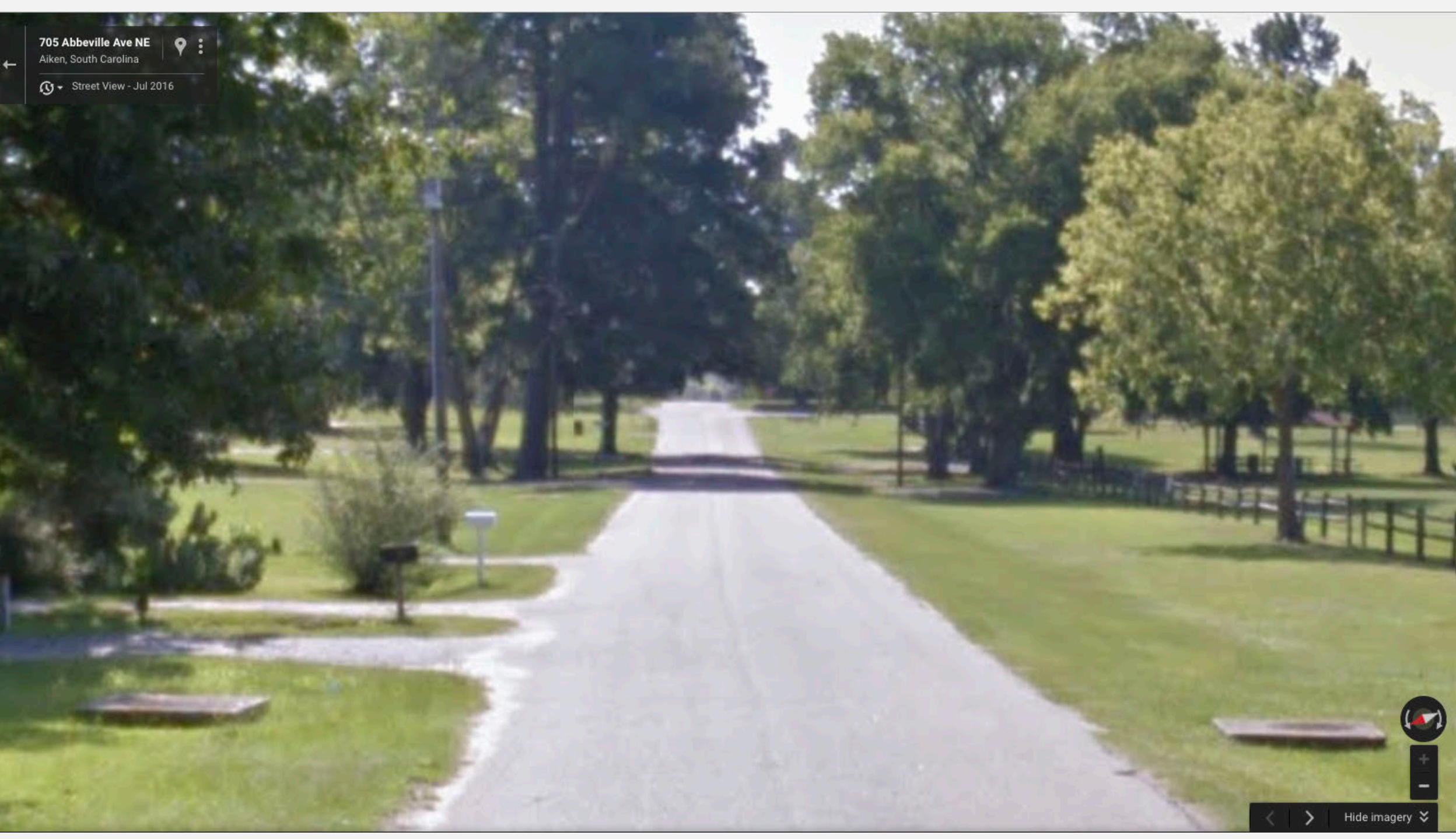

Streetview along Abbyville Ave, on bay floor looking at rim 240 meters away 


\section{Trenton, SC}

ansem 1.

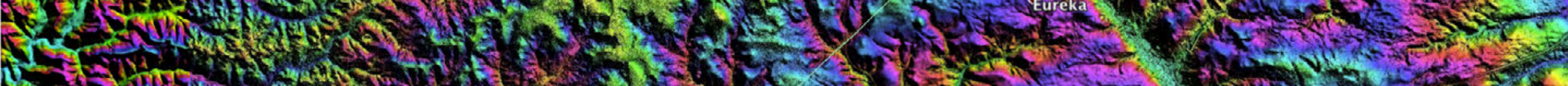
H.t. (1) Bat

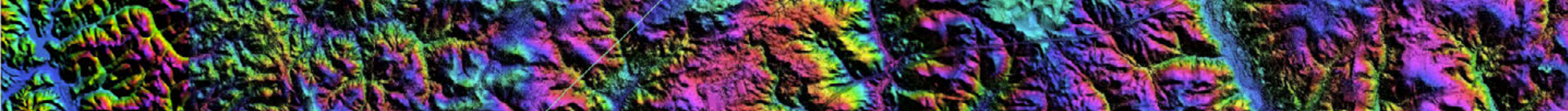
1.

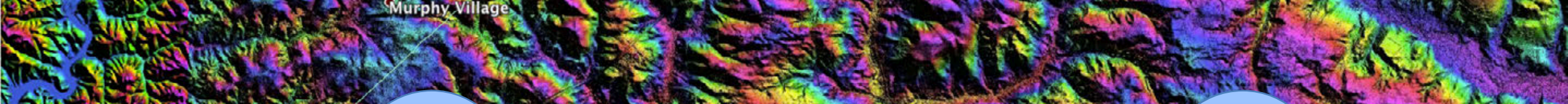

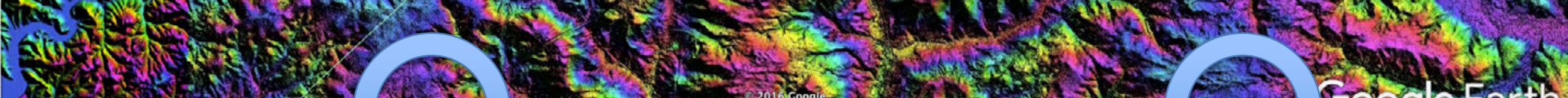

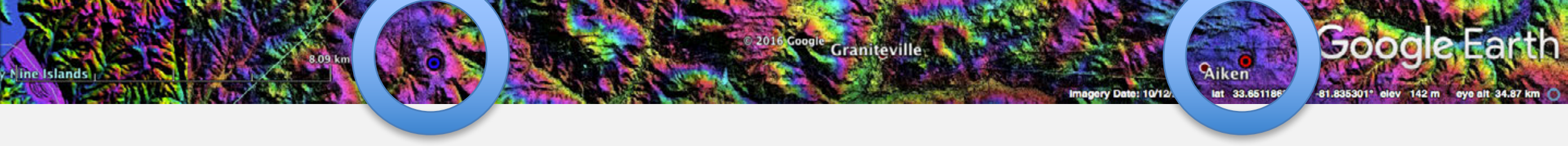

Bay near Trenton, $23 \mathrm{~km}$ NNW, with a $20 \mathrm{~m}$ rise in elevation 


\section{Trenton, SC}

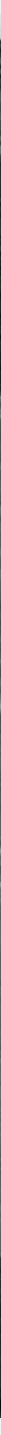

Bay near Trenton, $23 \mathrm{~km}$ NNW, with a $20 \mathrm{~m}$ rise in elevation 


\section{Trenton Area Bays}

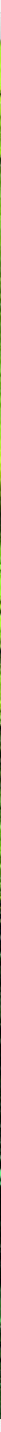

Headward erosion compromising nearby bays 


\section{Bay 135327_0810}

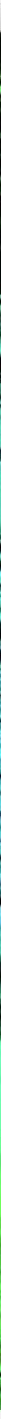

Major axis: $0.7 \mathrm{~km} \quad$ Bearing: 158으 Elevation: $183 \mathrm{~m}$ 
Bay 135327_1834

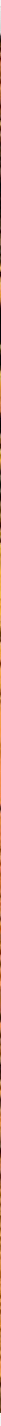

Major axis: $0.45 \mathrm{~km} \quad$ Bearing: 154으 Elevation: $197 \mathrm{~m}$ 


\section{Bay 135327_3511}

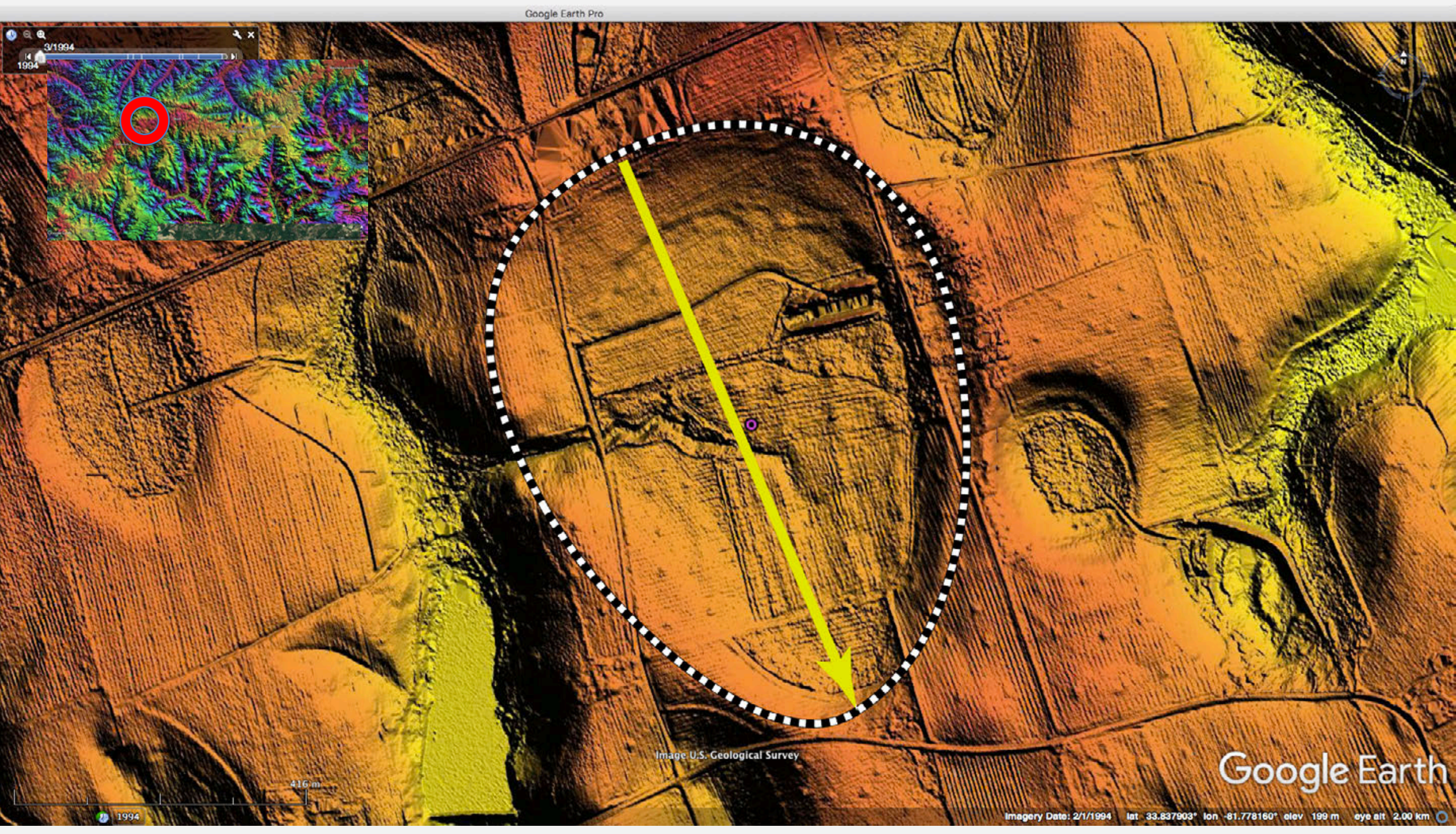

Major axis: $0.89 \mathrm{~km} \quad$ Bearing: 159을 Elevation: $201 \mathrm{~m}$ 


\section{Aggrading or Degrading?}

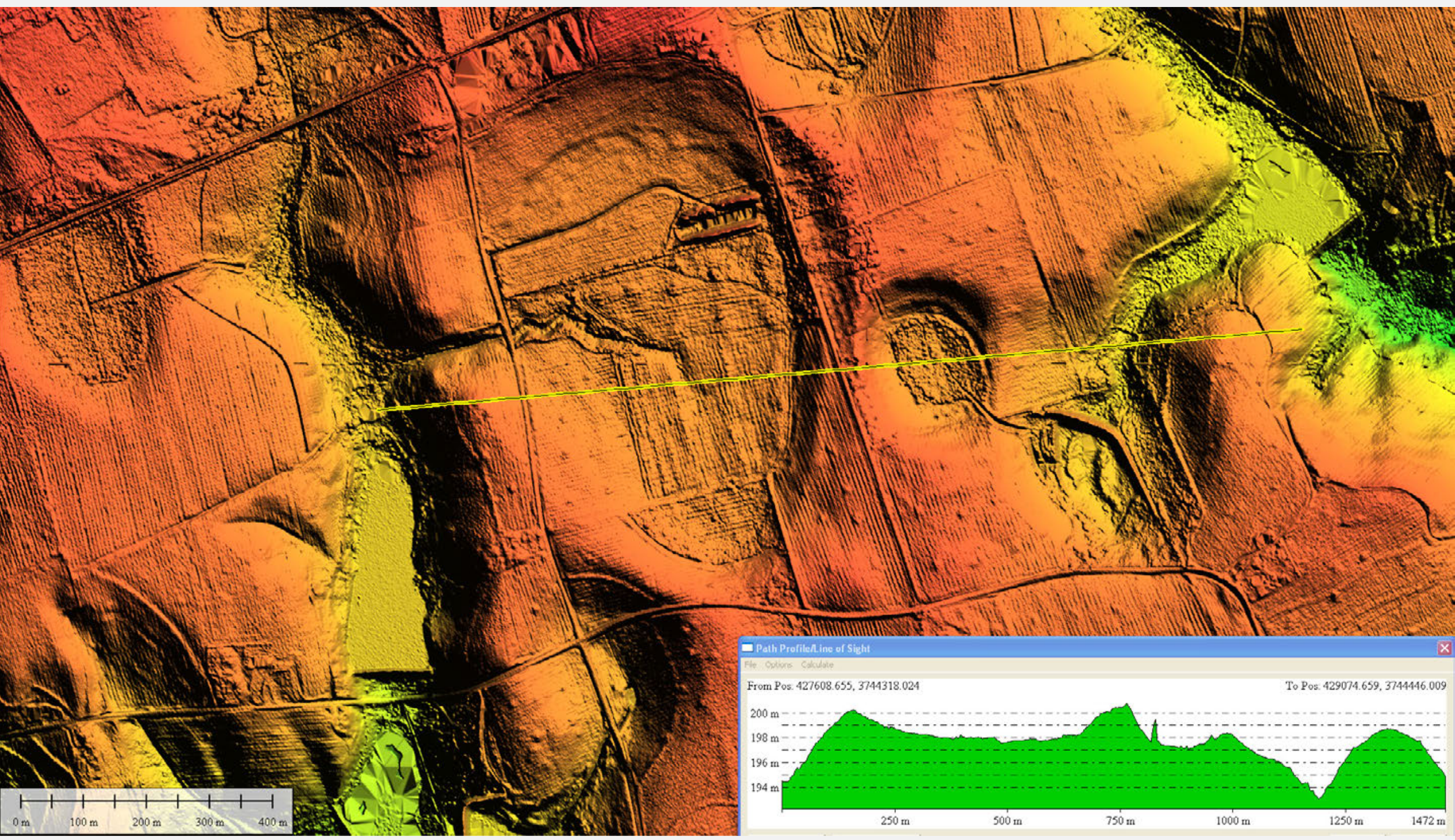

6 meters of relief across $1.5 \mathrm{~km}$ 


\section{Bays in Johnston, SC Area}

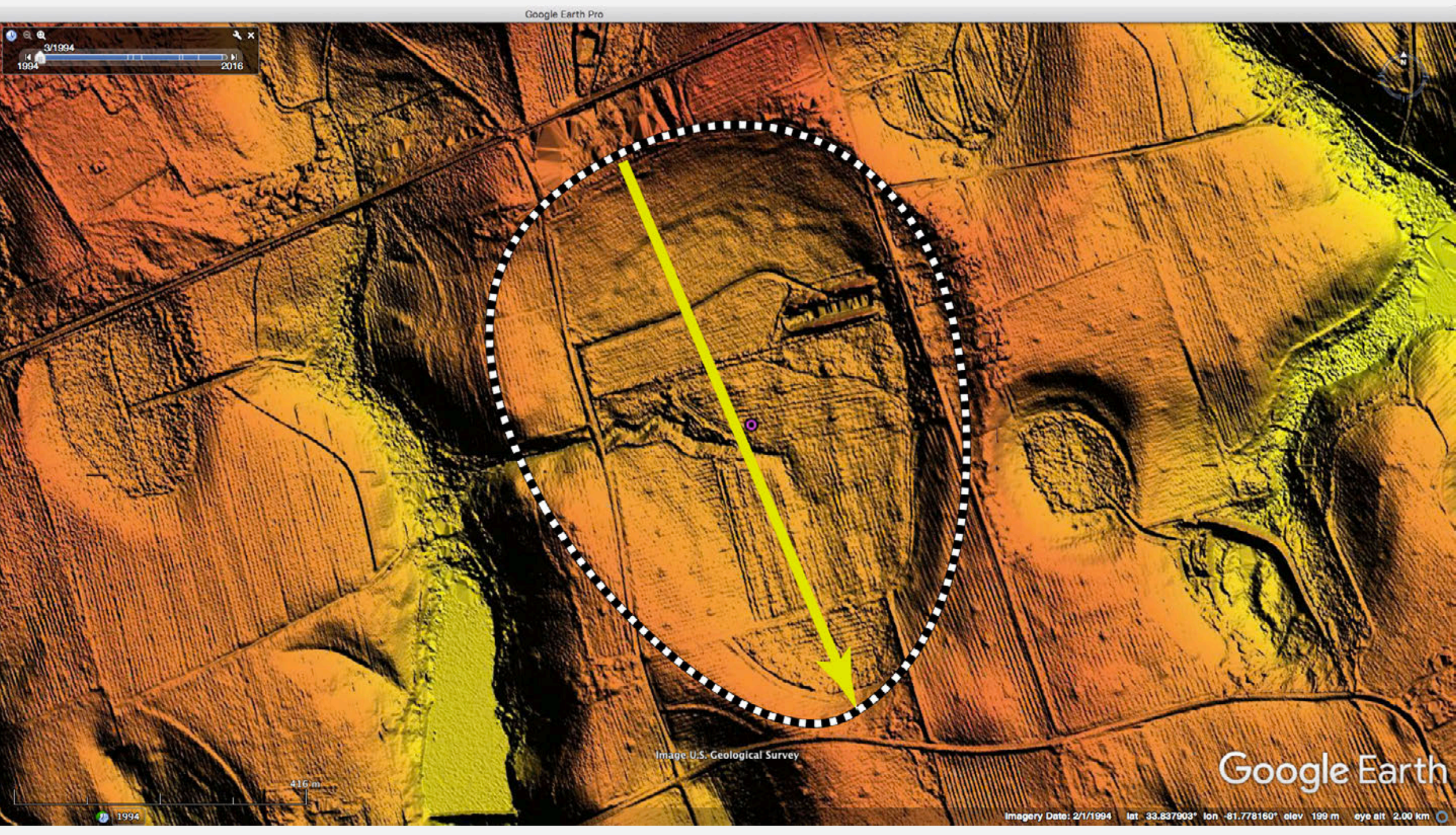

Please reference movie to view cookie-cutter in action: https://gsa.confex.com/gsa/2017SE/webprogram/Handout/Paper291016/JohnstonBays.pdf 
Bay 135326_3977

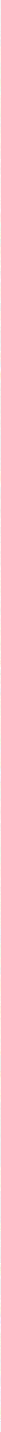

Major axis: $0.68 \mathrm{~km} \quad$ Bearing: 161으 Elevation: $194 \mathrm{~m}$ 
Bay 135326_3957

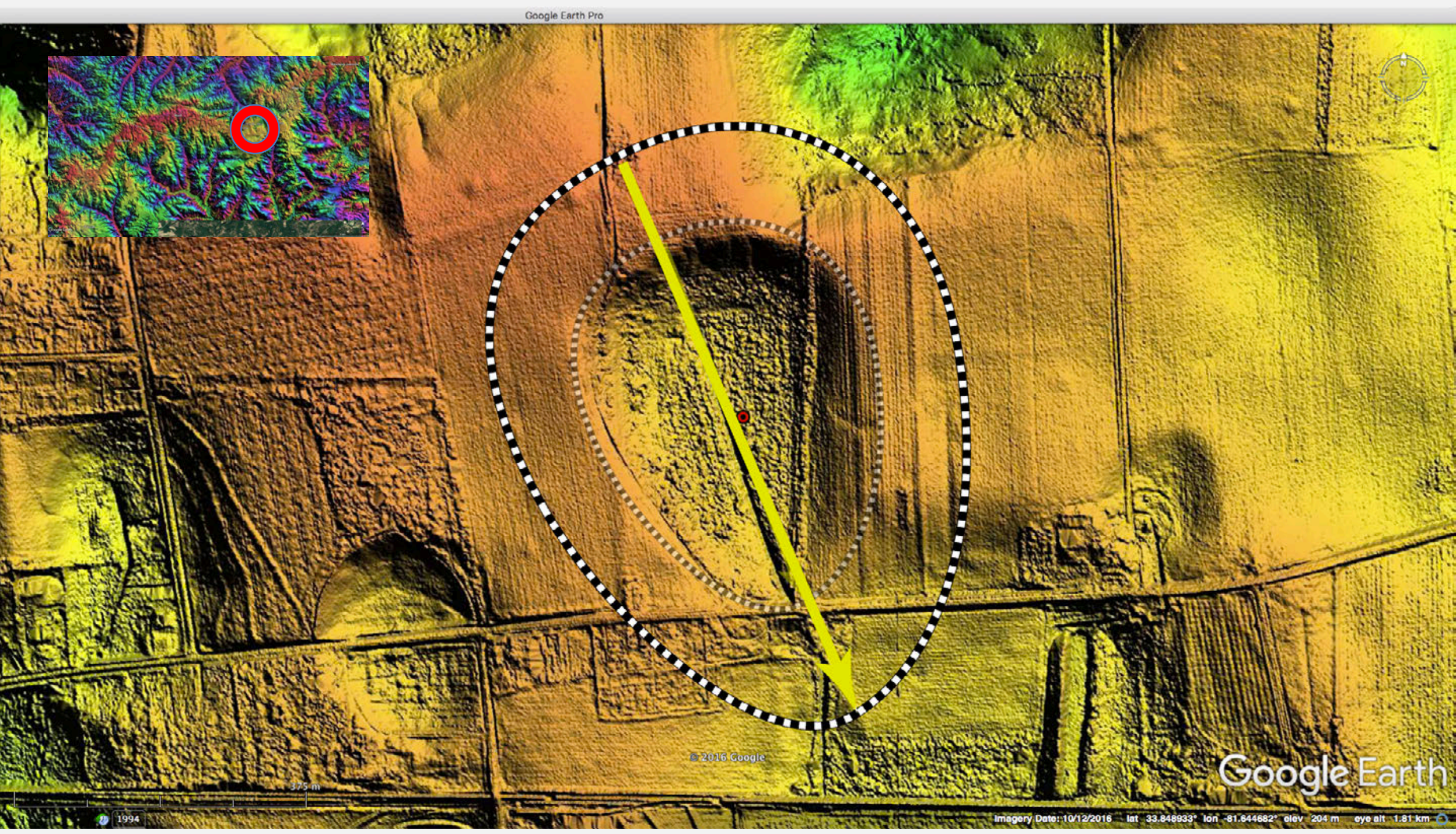

Major axis: $0.5 \mathrm{~km} \quad$ Bearing: 158을 $192 \mathrm{~m}$ 


\section{Bay 135326_3756 viewed from 2700 m}

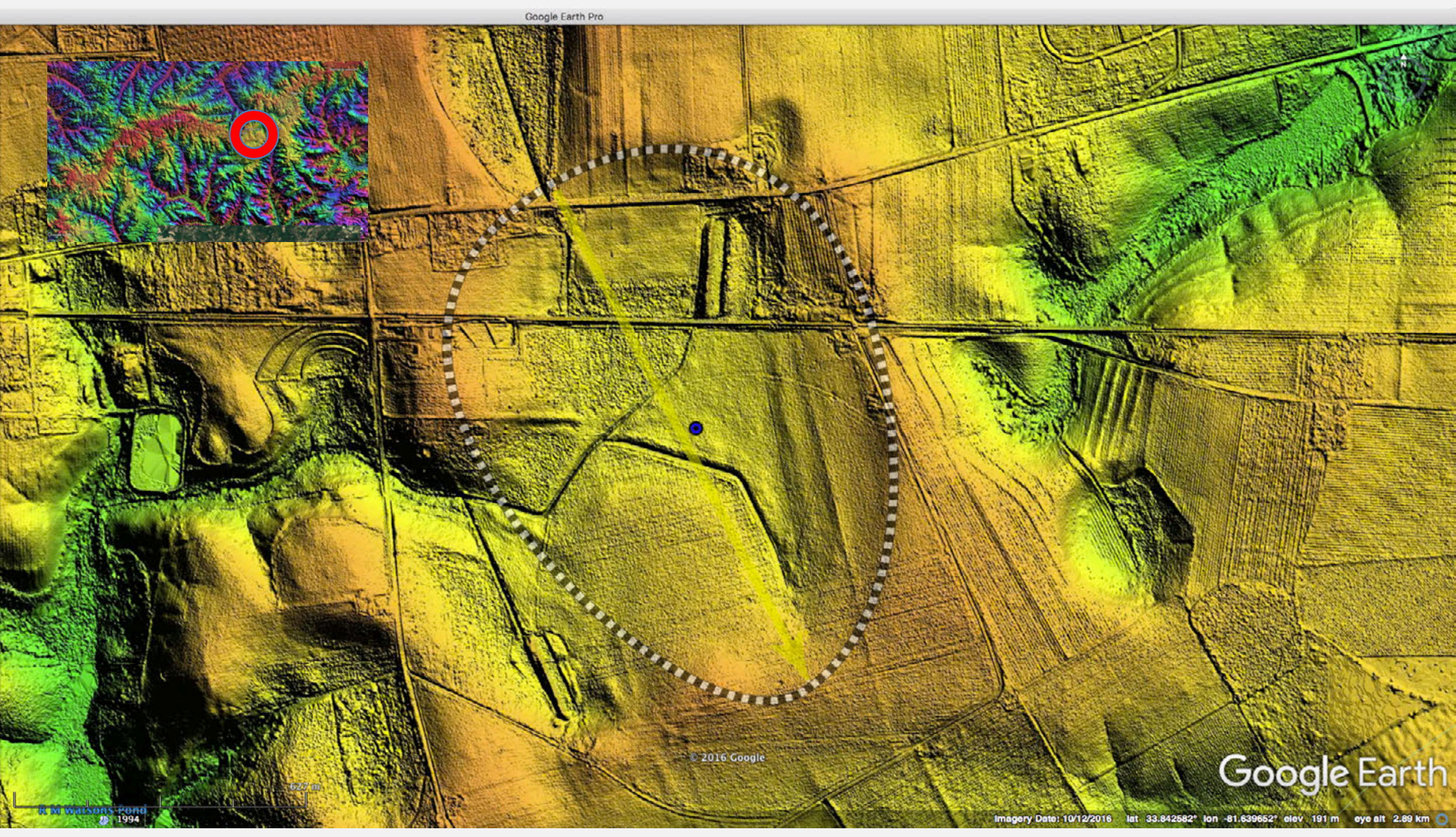

Major axis: $1.22 \mathrm{~km} \quad$ Bearing: 153으 Elevation: $190 \mathrm{~m}$ 


\section{Bay 135326_6405}

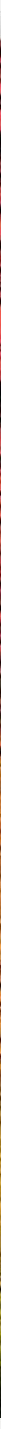

Major axis: $0.49 \mathrm{~km} \quad$ Bearing: 156을 Elevation: $196 \mathrm{~m}$ 


\section{Bay 135326_6405}

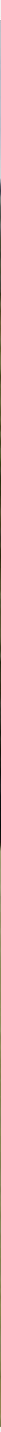

Streetview to West along Hampton Terrace, on bay floor looking at rim 150 meters away 


\section{Carolina Bays of Ridge Spring}

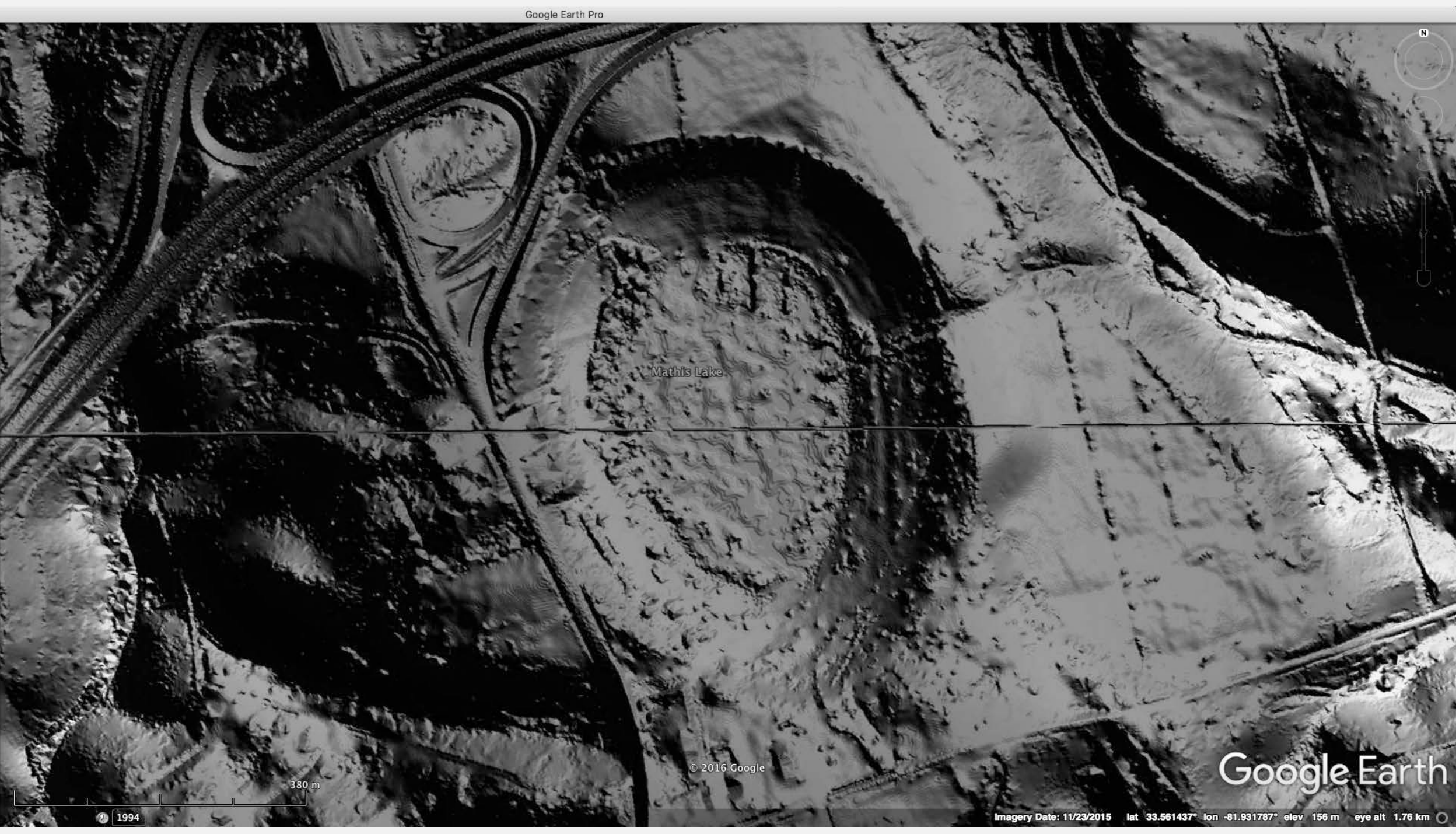

Grey scale comparison, image normalized for size 


\section{Carolina Bays of Ridge Spring}

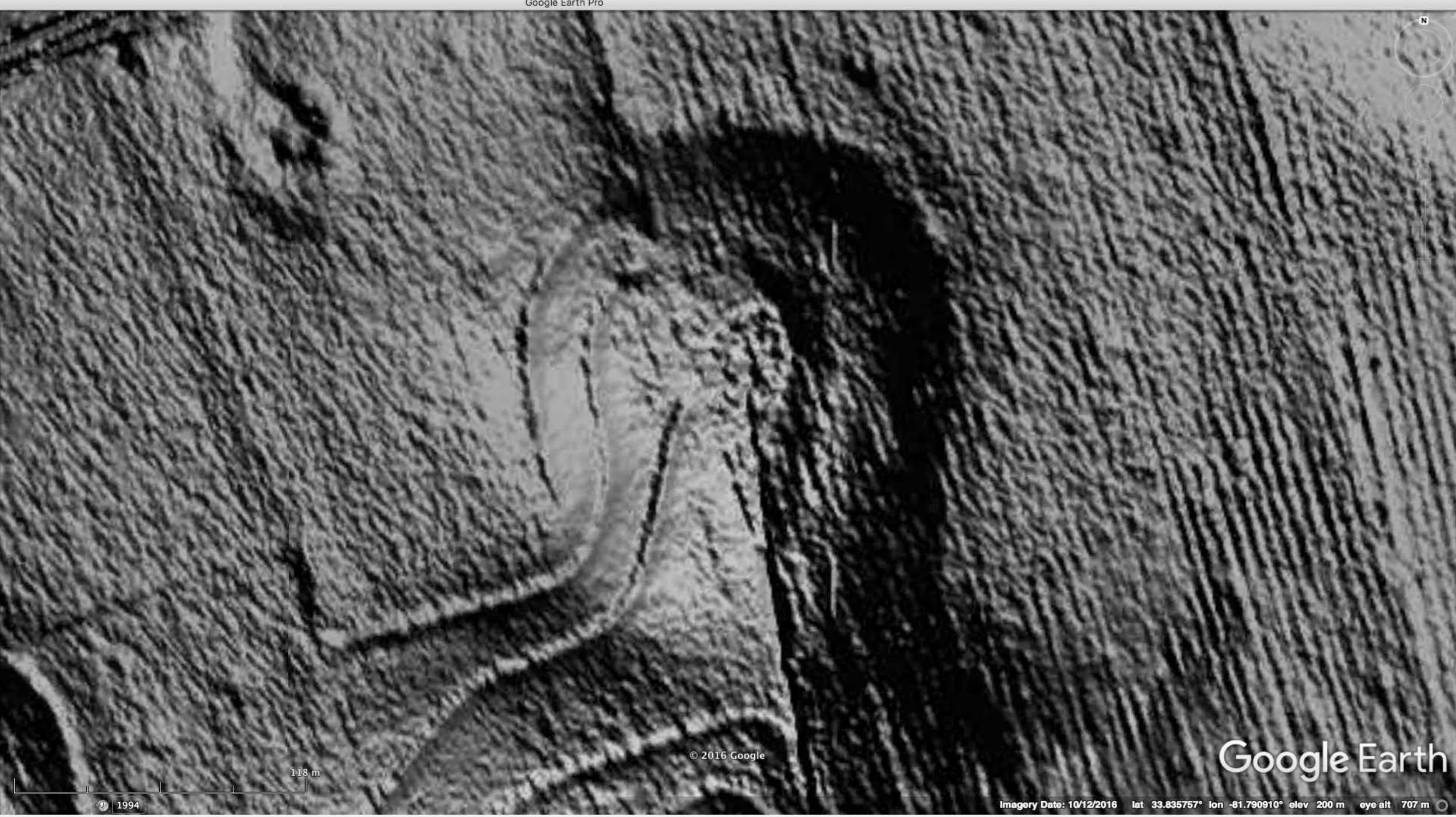

Grey scale comparison, image normalized for size 


\section{Carolina Bays of Ridge Spring}

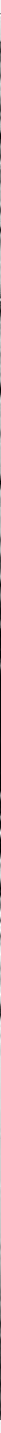

Grey scale comparison, image normalized for size 


\section{Carolina Bays of Ridge Spring}

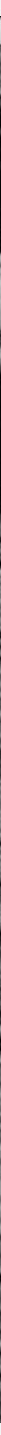

Grey scale comparison, image normalized for size 


\section{Carolina Bays of Ridge Spring}

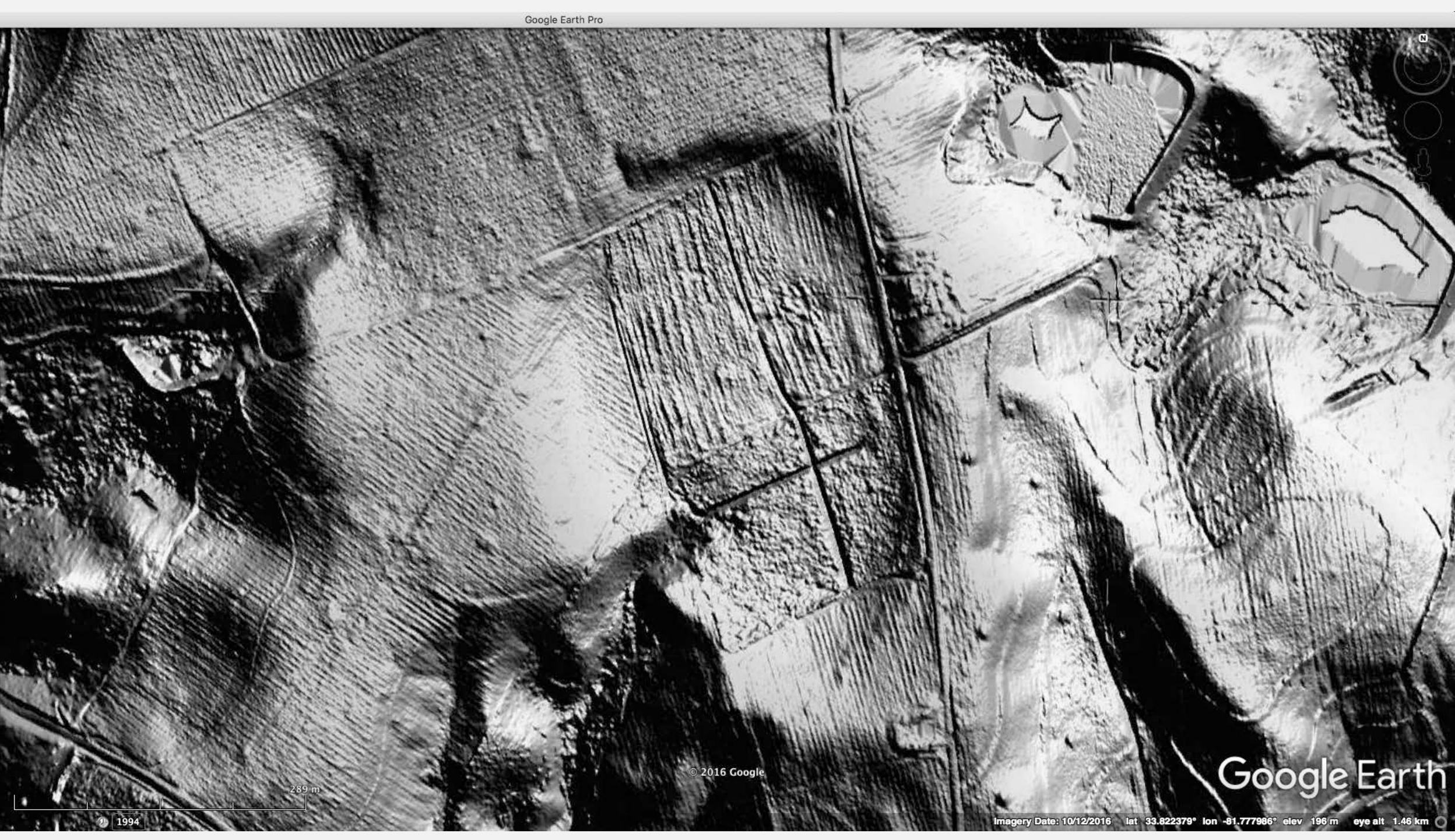

Grey scale comparison, image normalized for size 


\section{Carolina Bays of Ridge Spring}

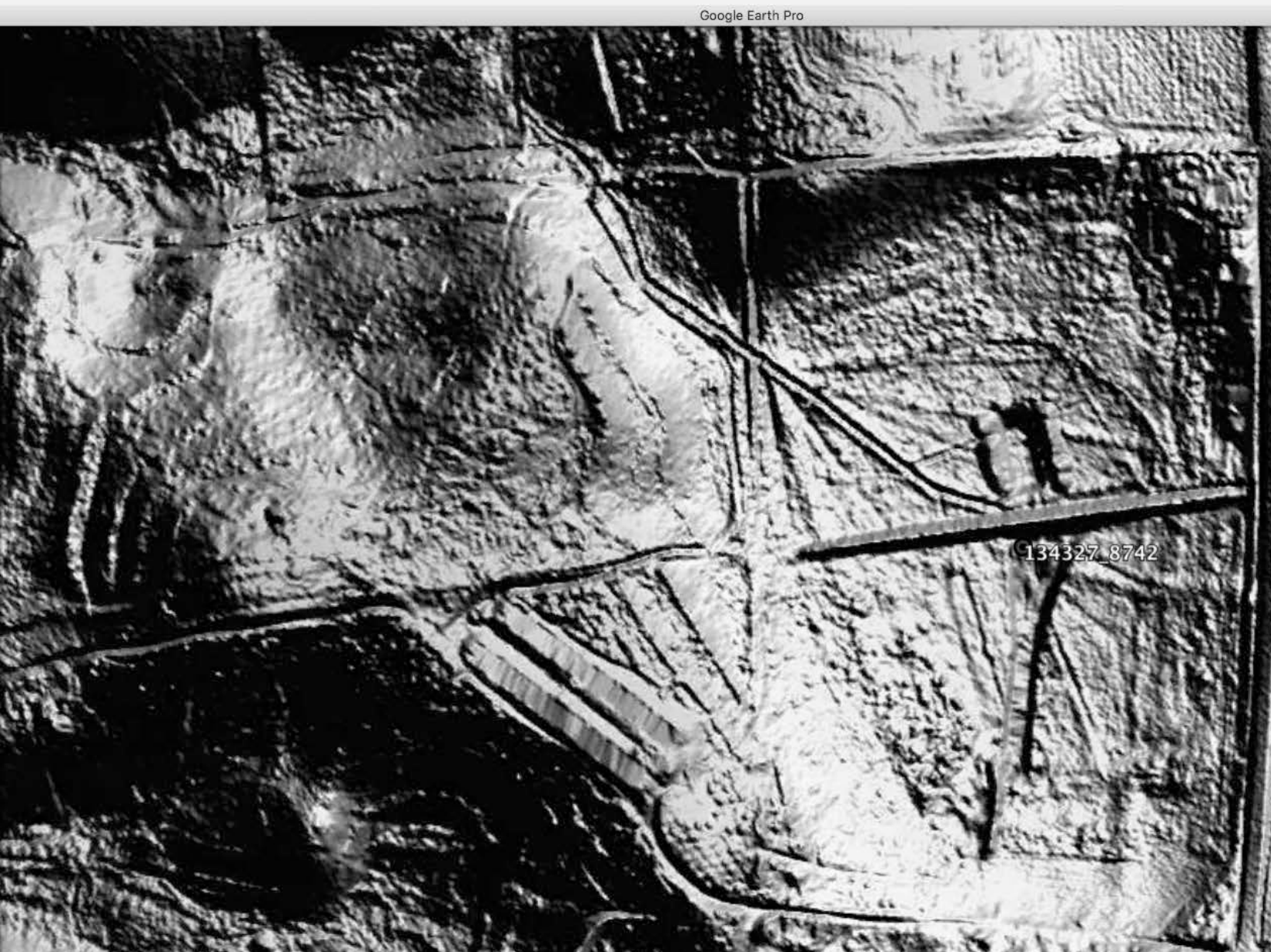

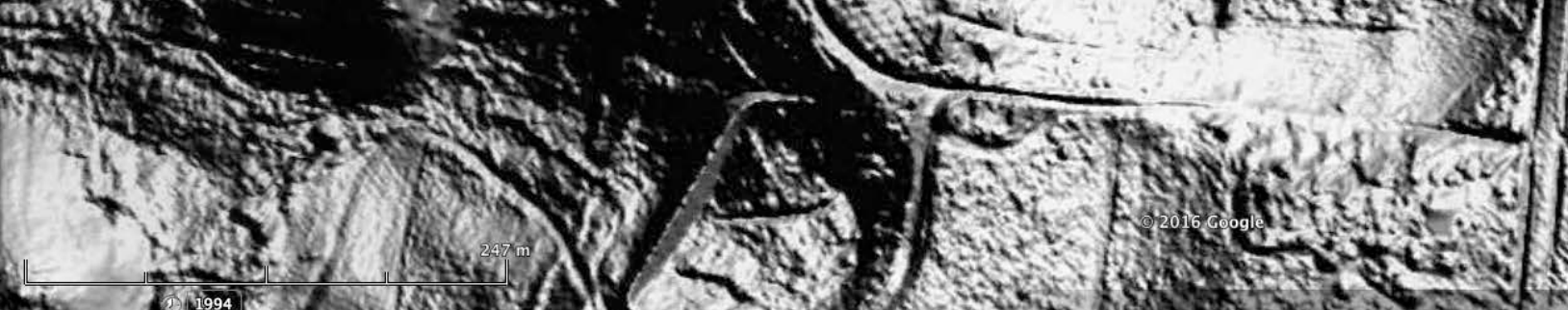

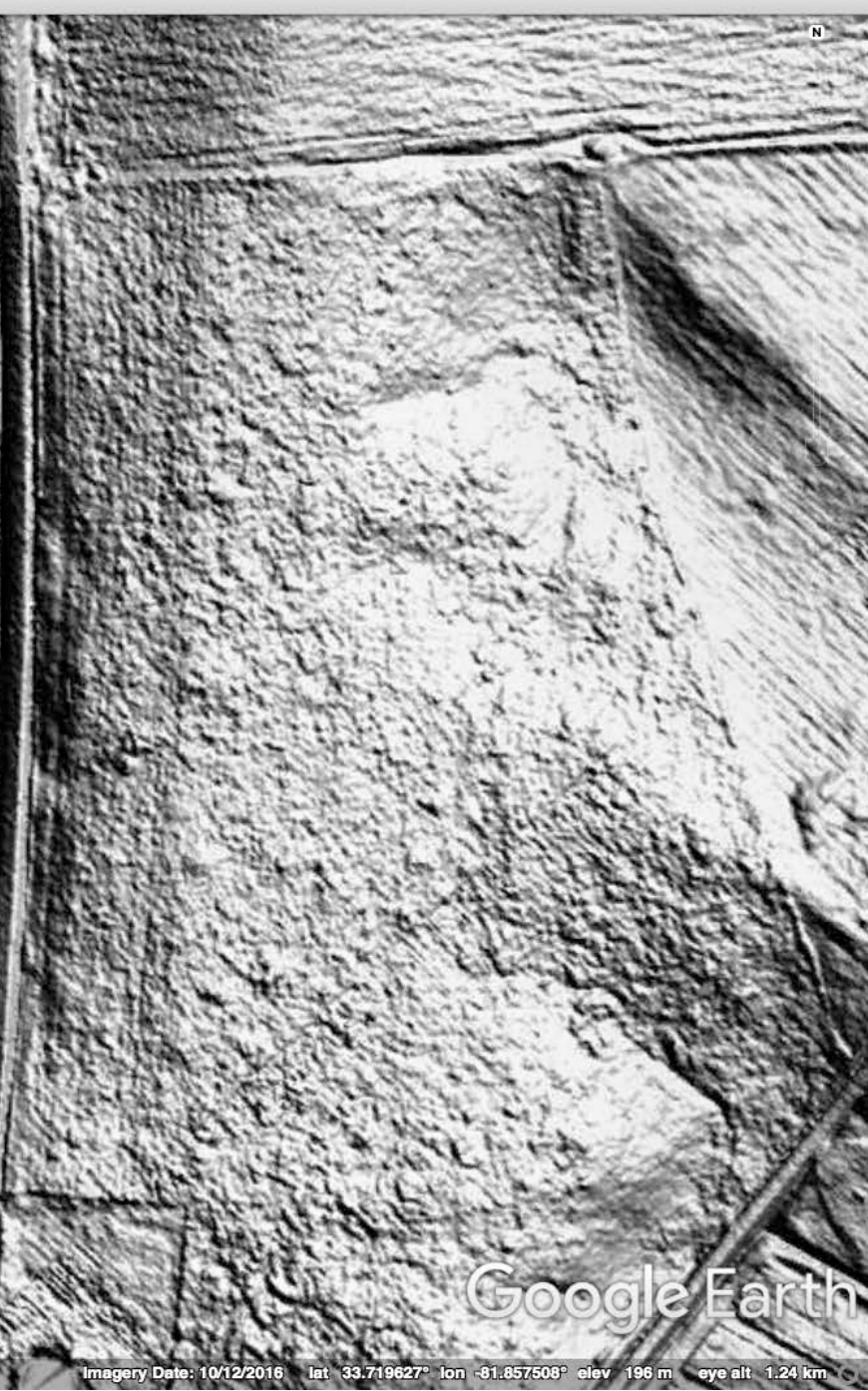

Grey scale comparison, image normalized for size 


\section{Carolina Bays of Ridge Spring}

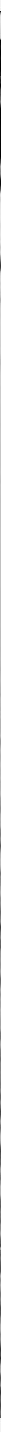

Grey scale comparison, image normalized for size 


\section{Carolina Bays of Ridge Spring}

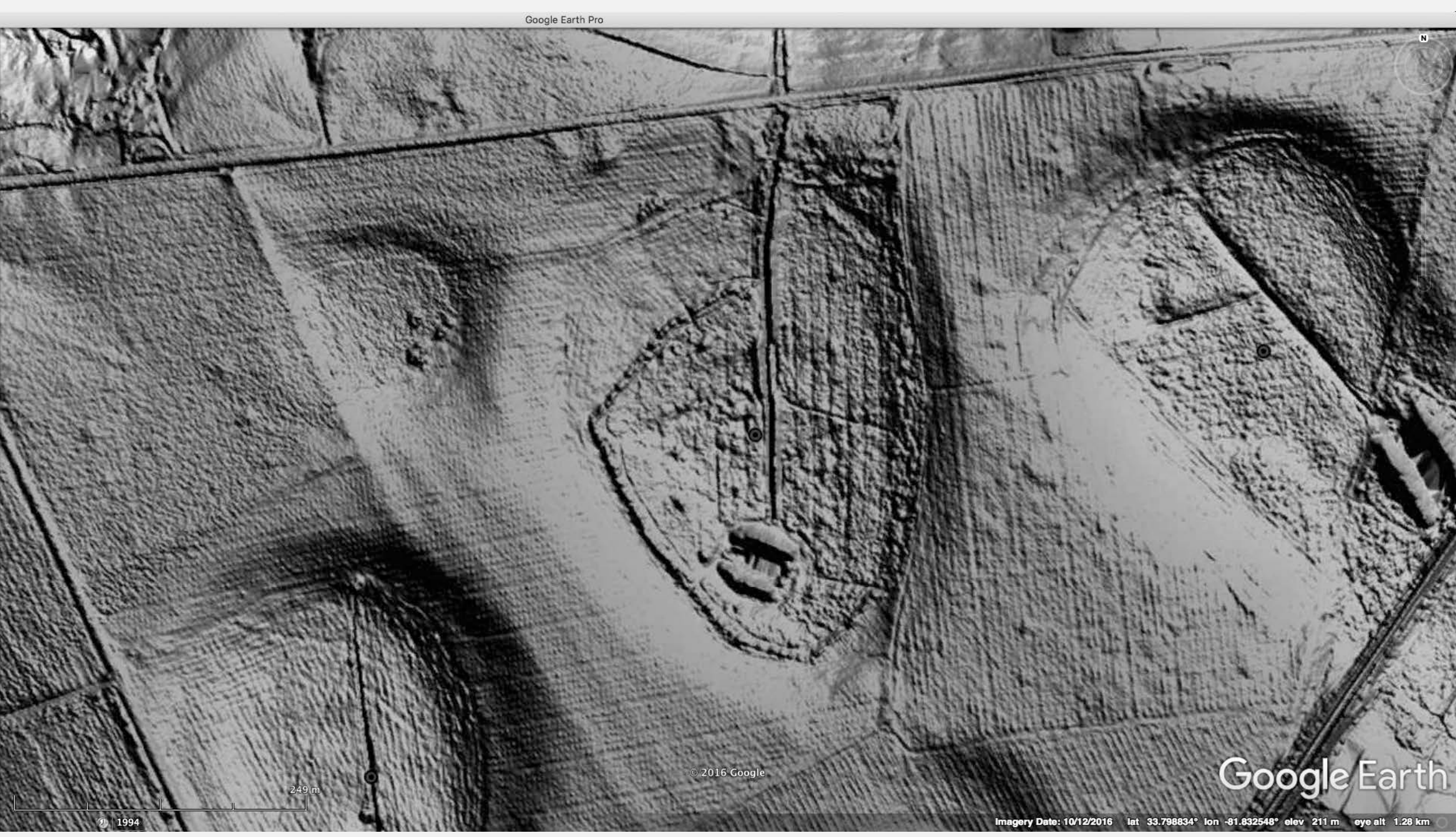

Grey scale comparison, image normalized for size 


\section{Carolina Bays of Ridge Spring}

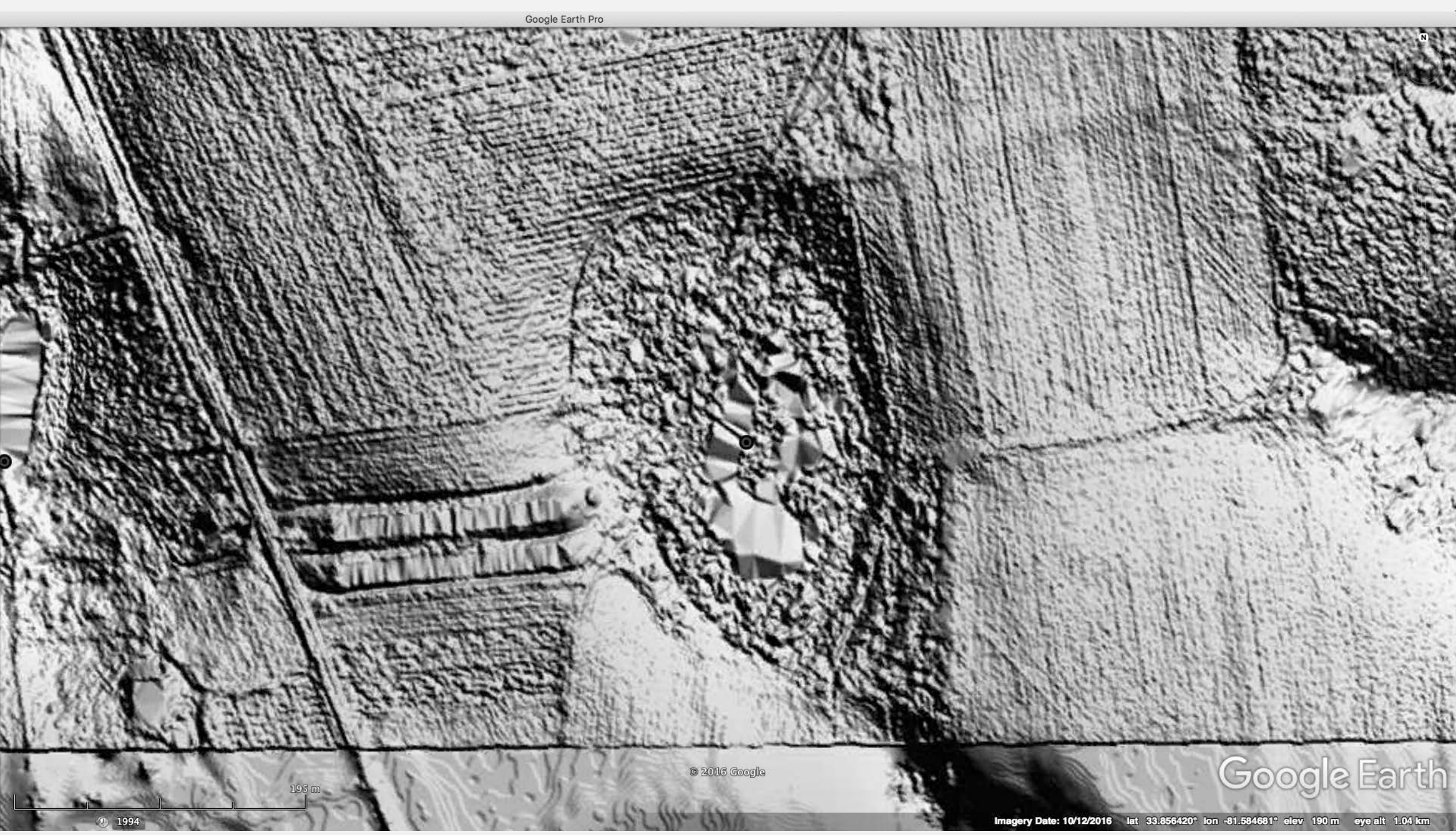

Grey scale comparison, image normalized for size 


\section{Carolina Bays of Ridge Spring}

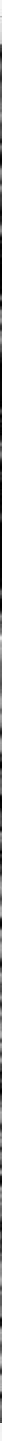

Grey scale comparison, image normalized for size 


\section{Carolina Bays of Ridge Spring}

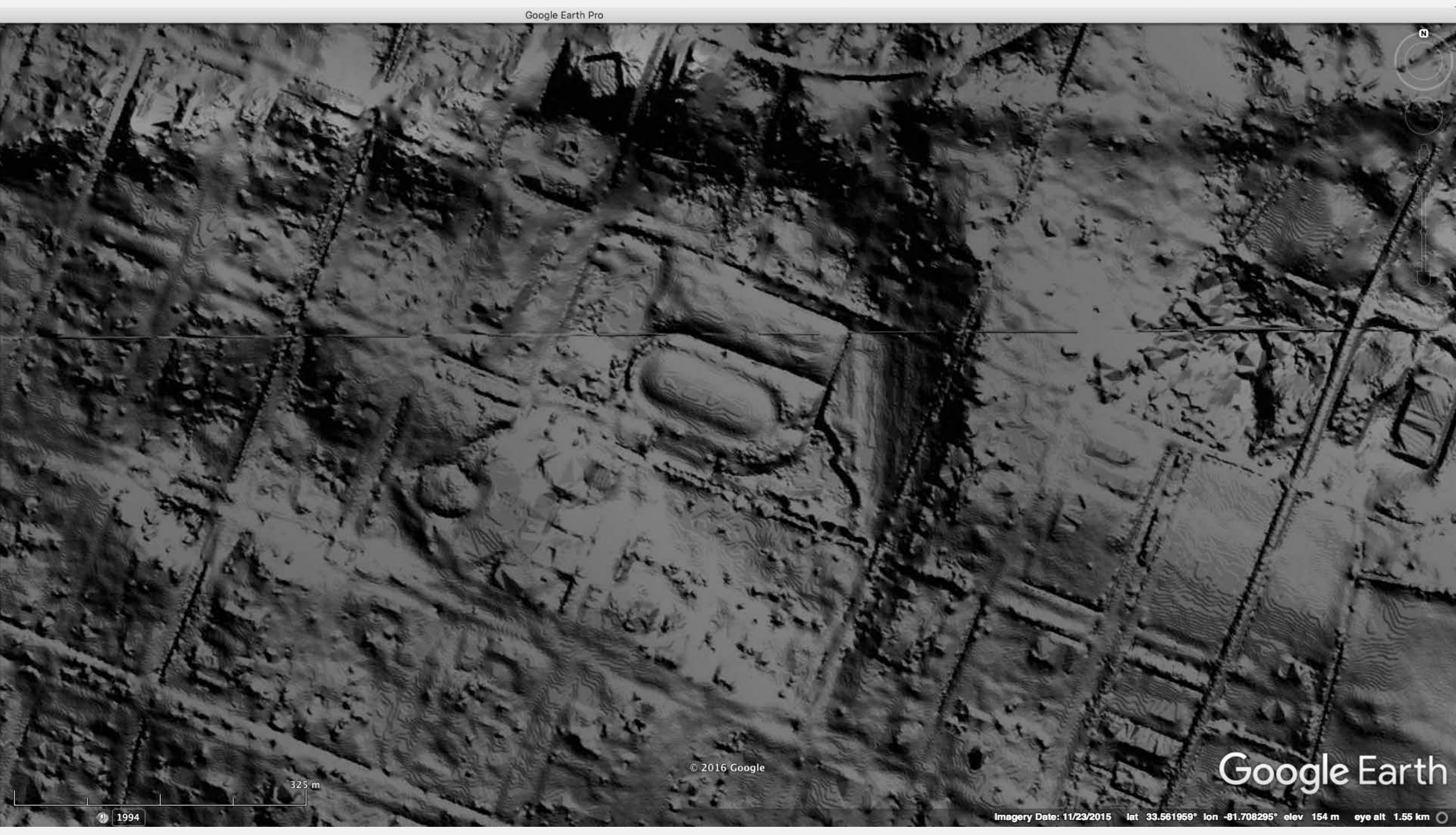

Grey scale comparison, image normalized for size 


\section{Carolina Bays of Ridge Spring}

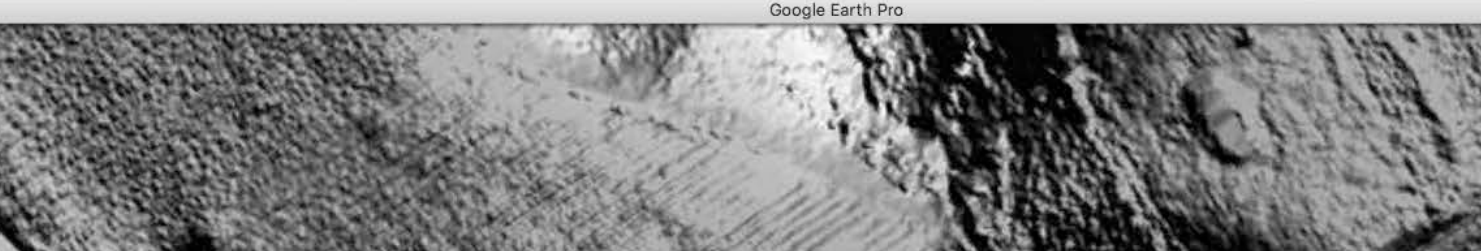

L

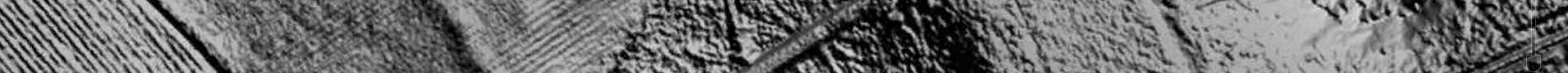
世
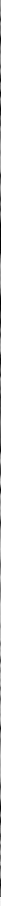


\section{Carolina Bays of Ridge Spring}

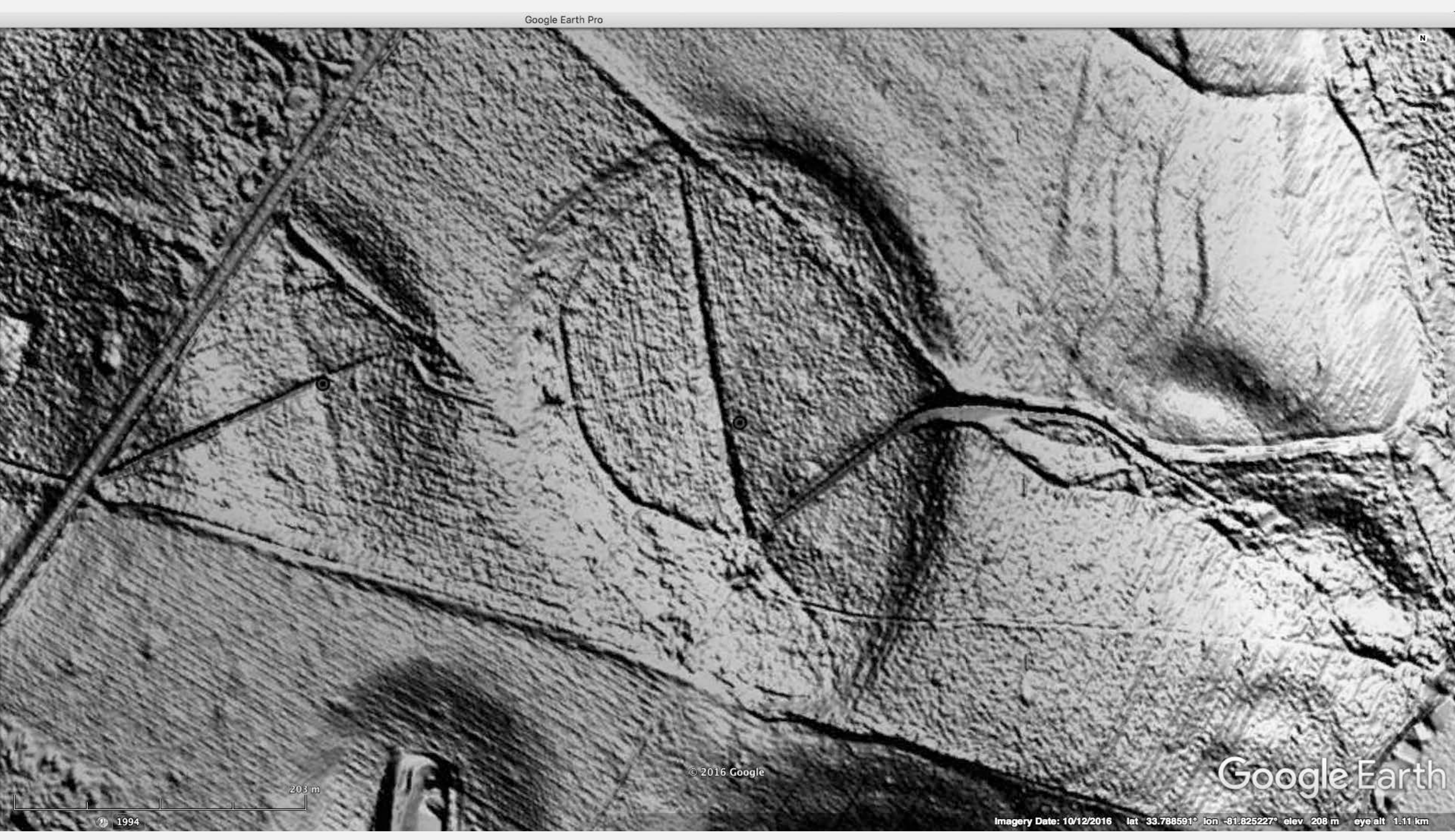

Grey scale comparison, image normalized for size 


\section{Carolina Bays of Ridge Spring}

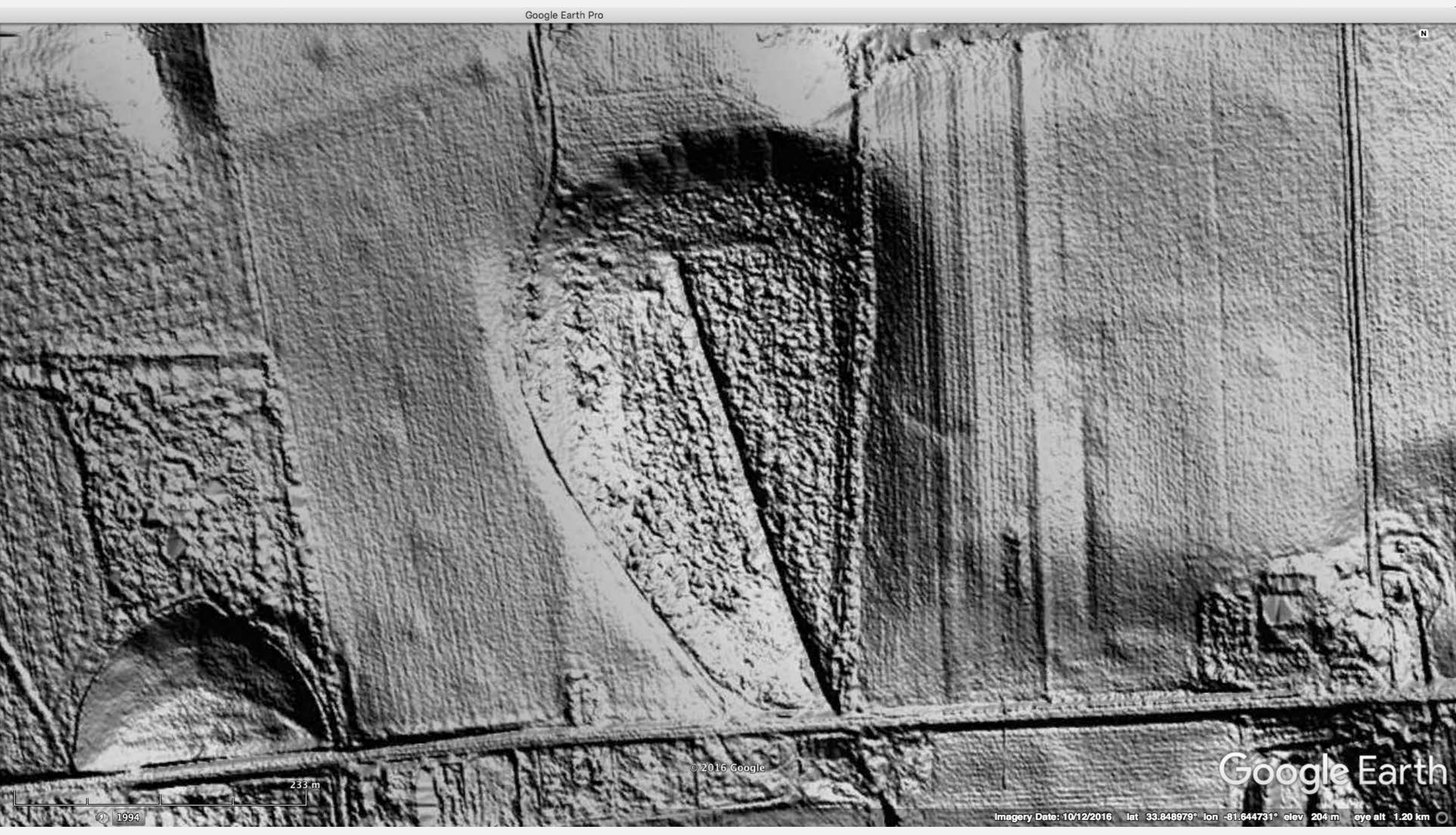

Grey scale comparison, image normalized for size 


\section{Carolina Bays of Ridge Spring}

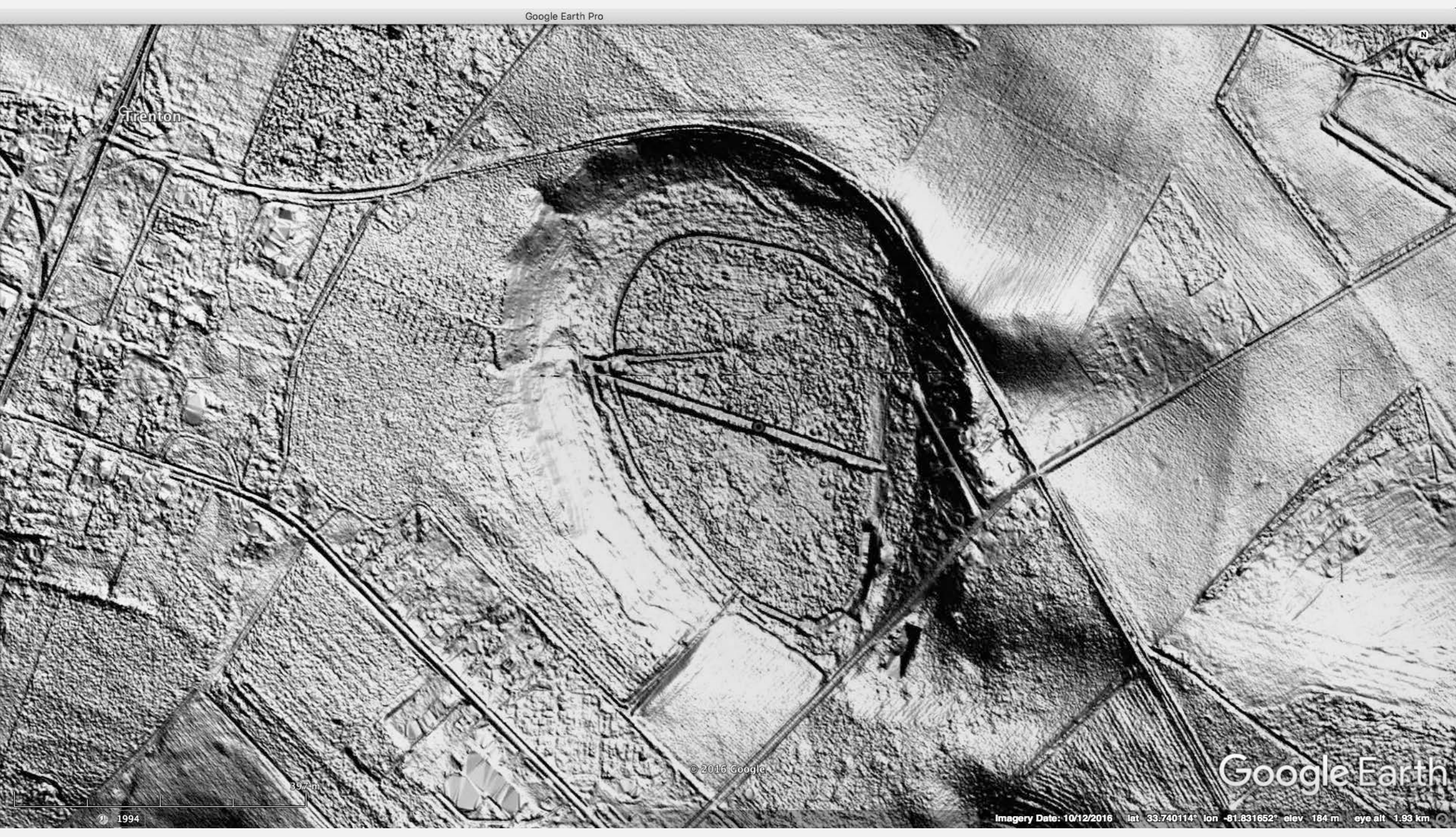

Grey scale comparison, image normalized for size 


\section{Carolina Bays of Ridge Spring}

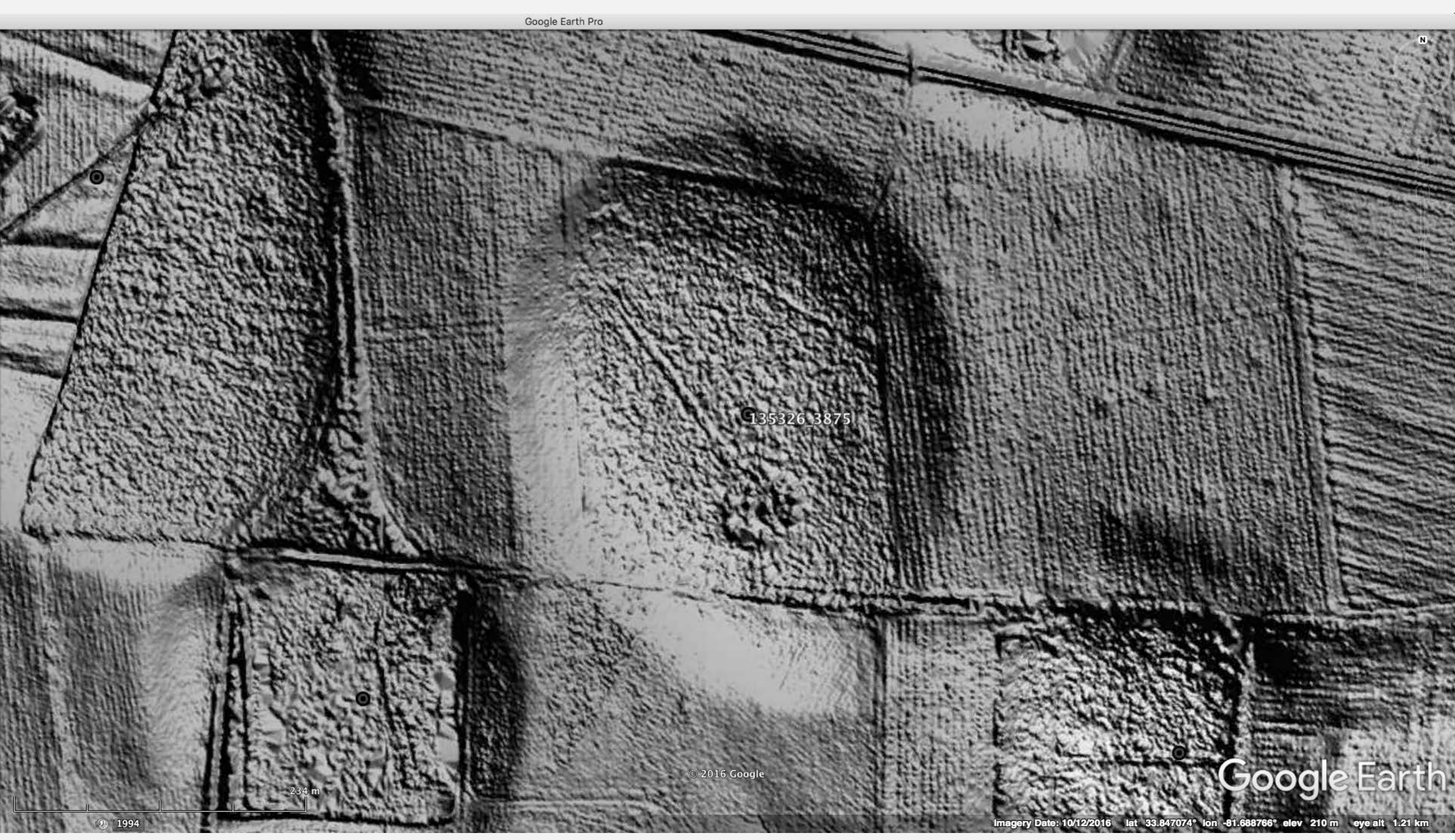

Grey scale comparison, image normalized for size 


\section{Carolina Bays of Ridge Spring}

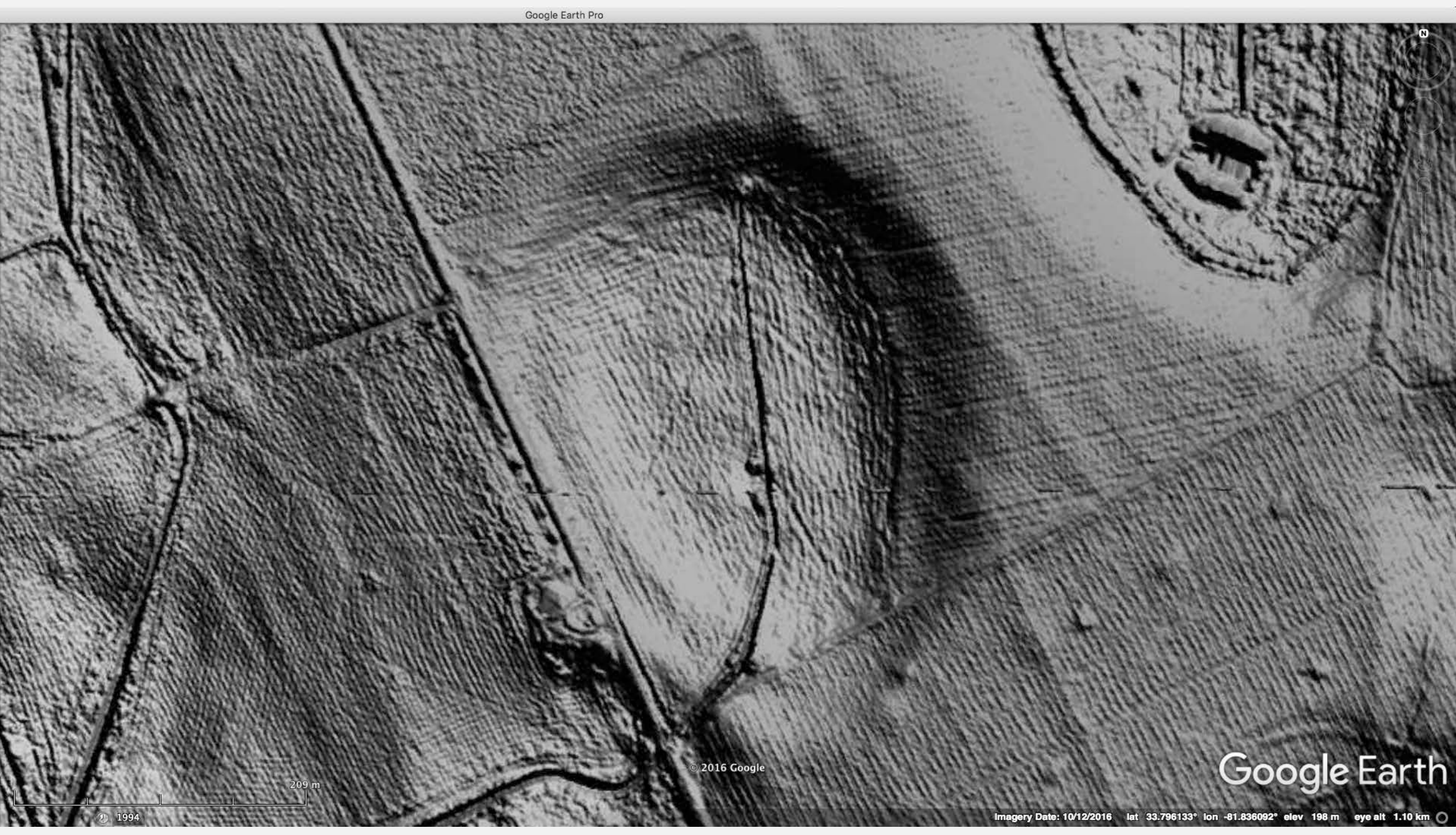

Grey scale comparison, image normalized for size 


\section{Carolina Bays of Ridge Spring}

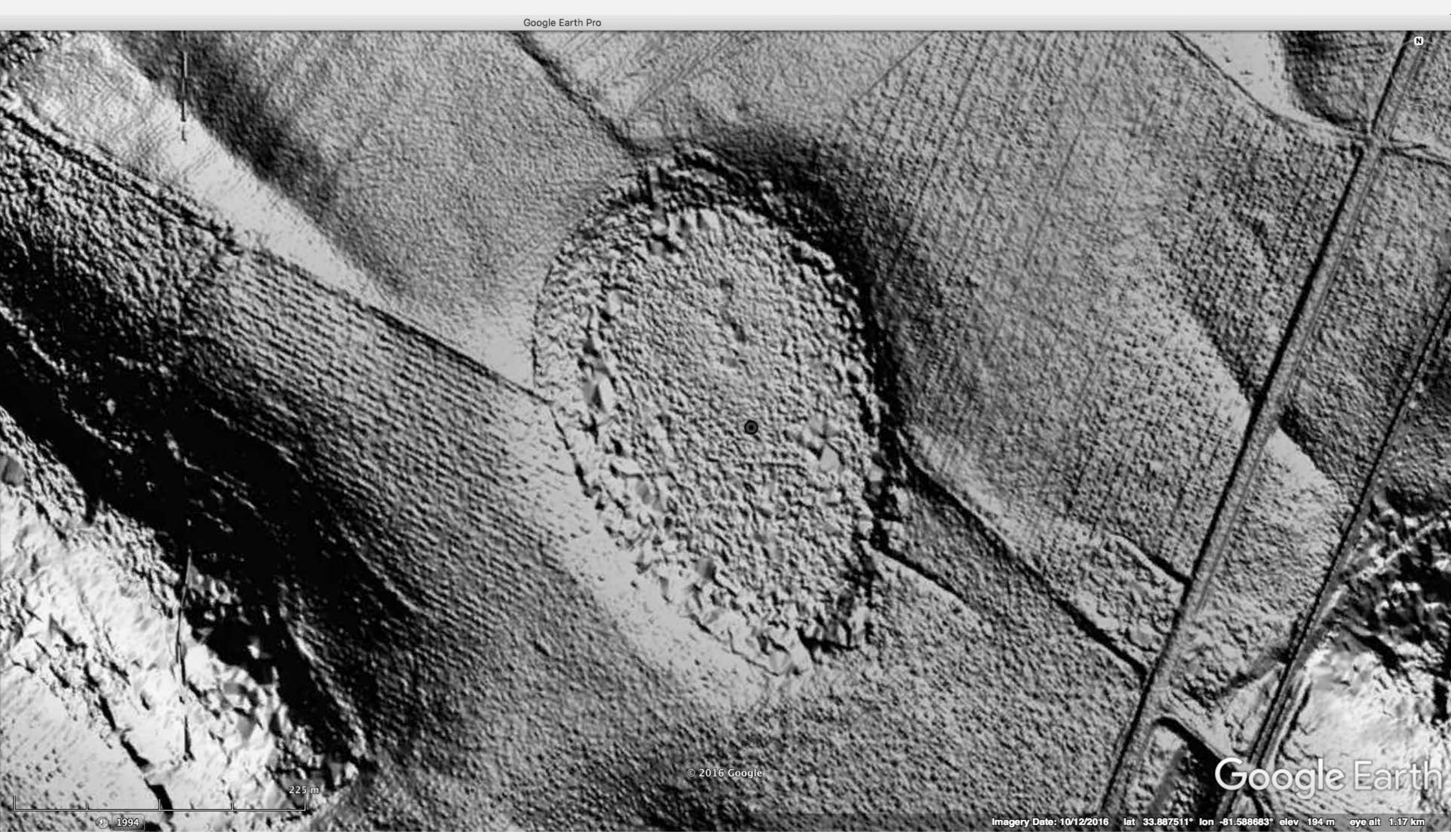

Grey scale comparison, image normalized for size 


\section{Carolina Bays of Ridge Spring}

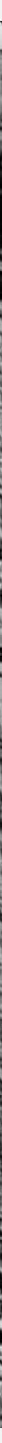

Grey scale comparison, image normalized for size 


\section{Carolina Bay Survey}

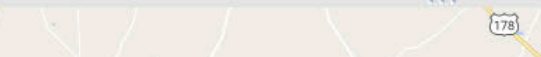

(2) एक्ष
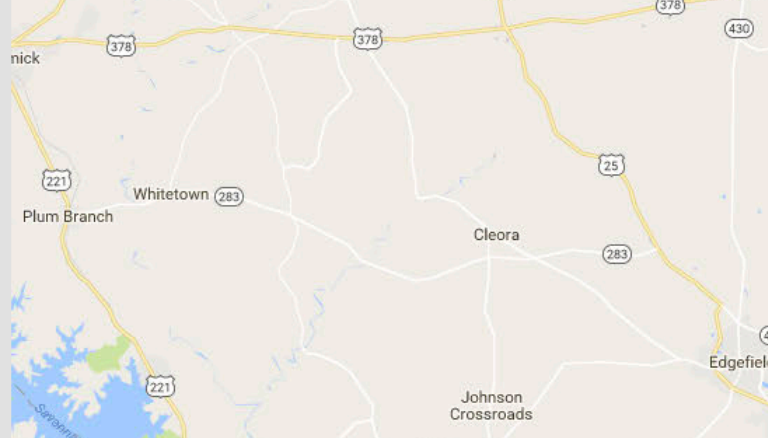

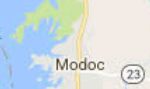

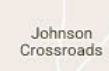

$$
\text { (23) }
$$
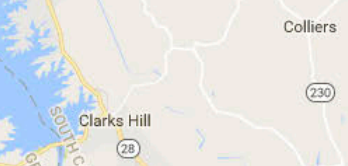

20

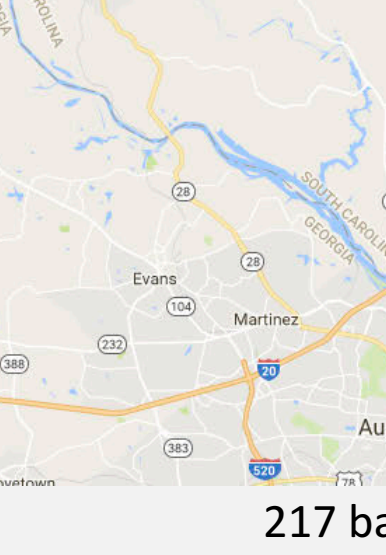

217 bays selected:<smiles>C1CC1</smiles>

(3) 


\section{Taxonomy of landform Genus "Carolina Bay"}

- Six tightly constrained archetypes as Species

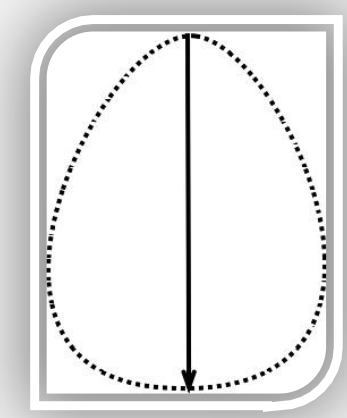

- Bell

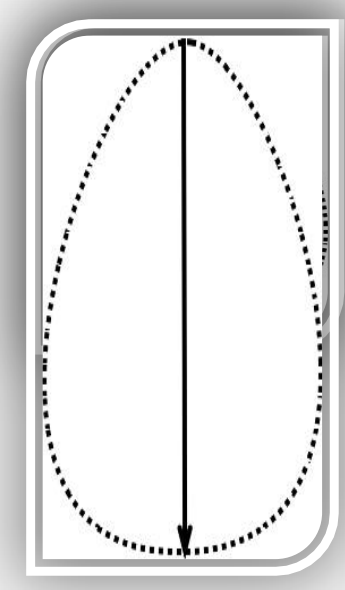

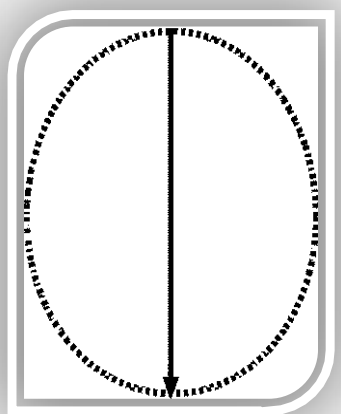

Oval

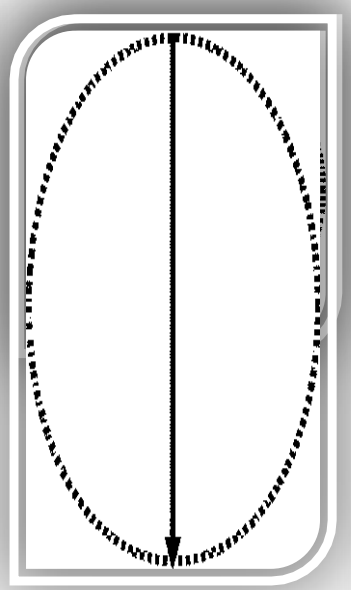

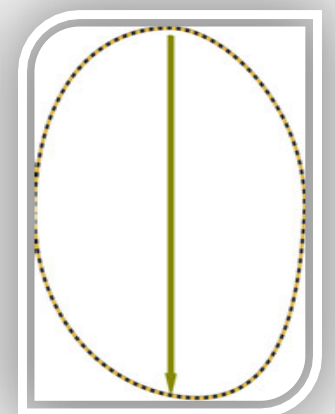

Shore

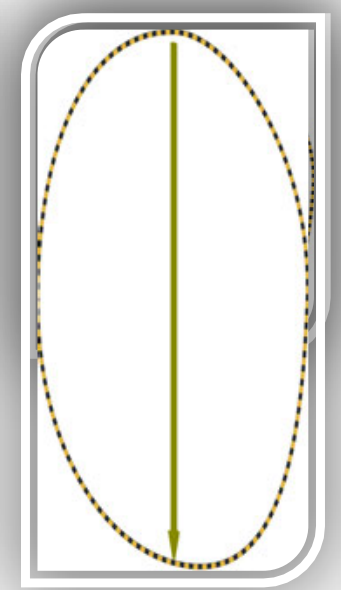

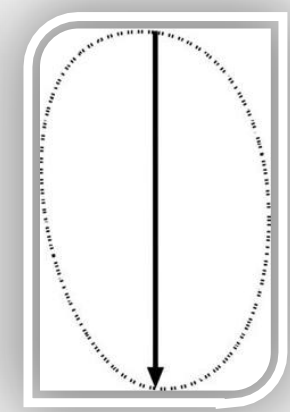

Carolina

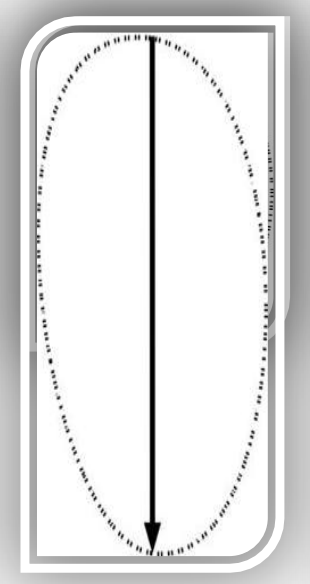

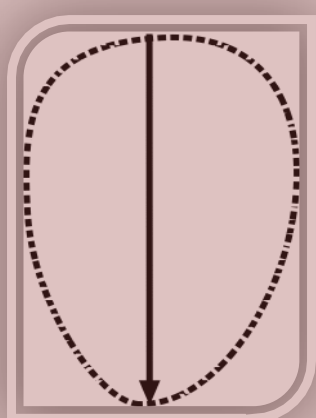

South

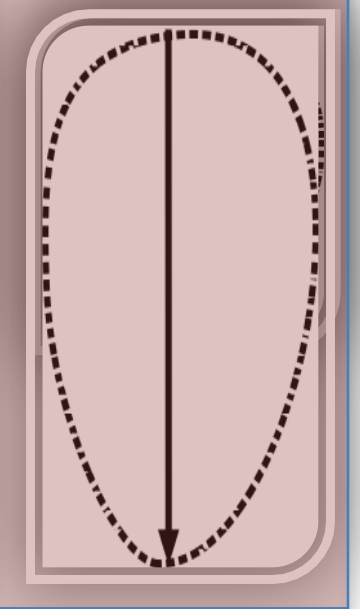

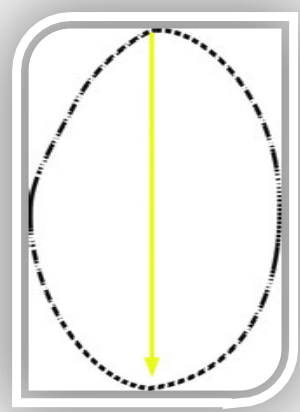

West

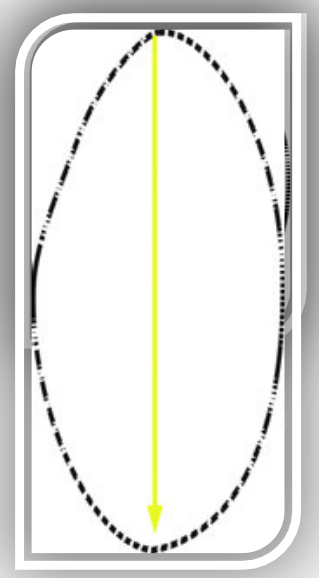




\section{Distribution of Bays by Planform Shape}

Category

Summarize data?

Value

- Count of Planform

Sum of Octant -

Sort by

count -

Maximum slices

10

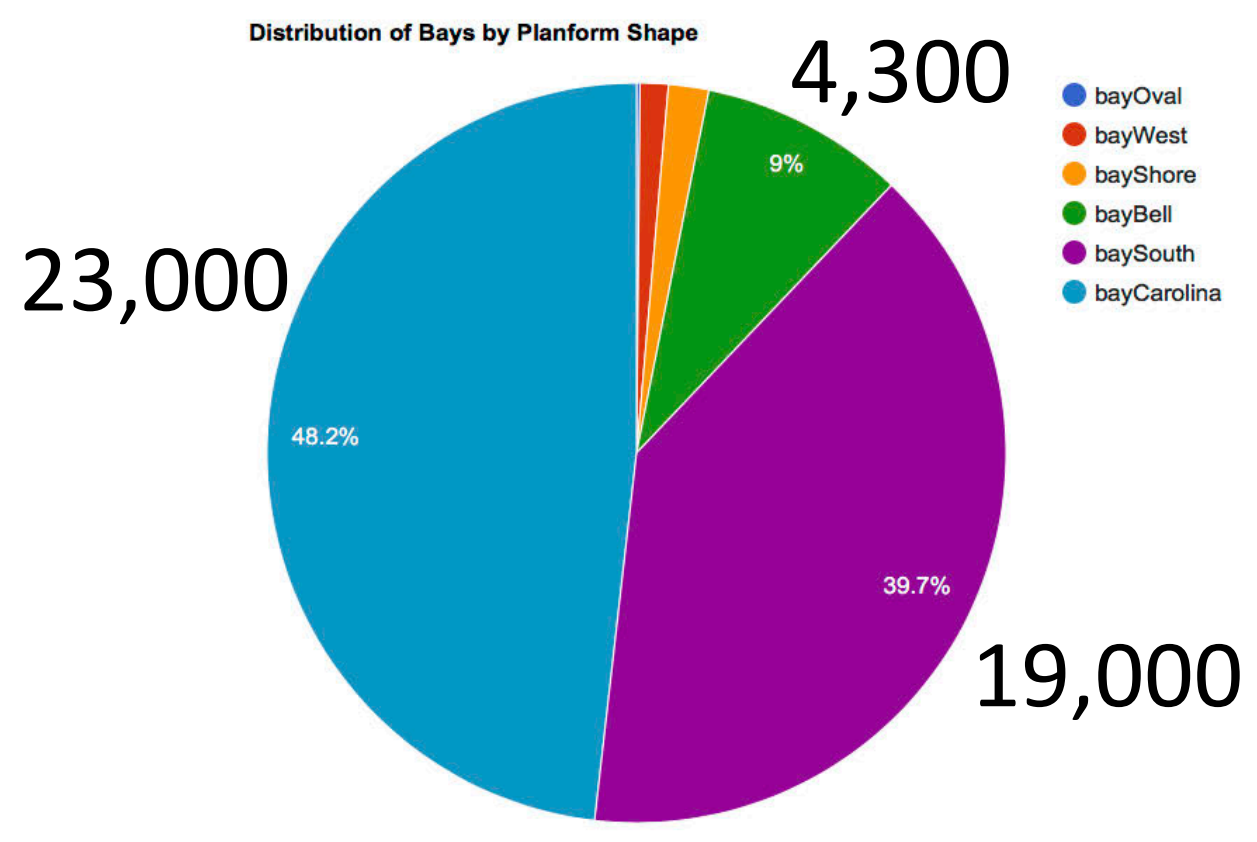

baySouth represents $\sim 40 \%$ of all 48,132 bays in Survey 


\section{0 - 125 m, 1121150 m Up, 372}

Ridge Spring, 217

50 - 75 m, 13949

$25-50 \mathrm{~m}, 10577$

\section{$25 \mathrm{~m}$ Buckets}




\section{Family Resemblance}

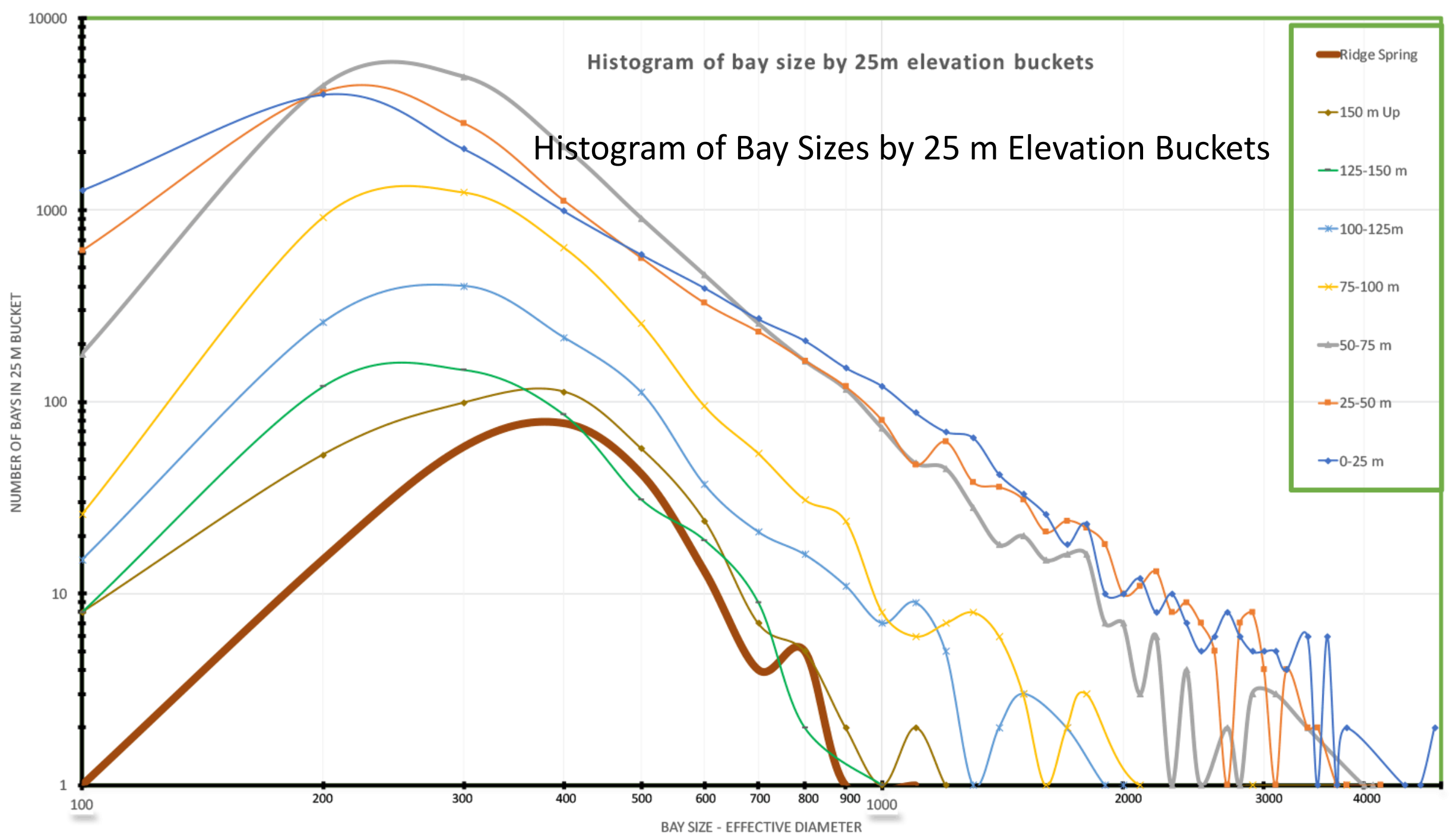

All Eastern bays compared to bays in Ridge Spring area 


\section{Heavily Eroded bayCarolina Species}
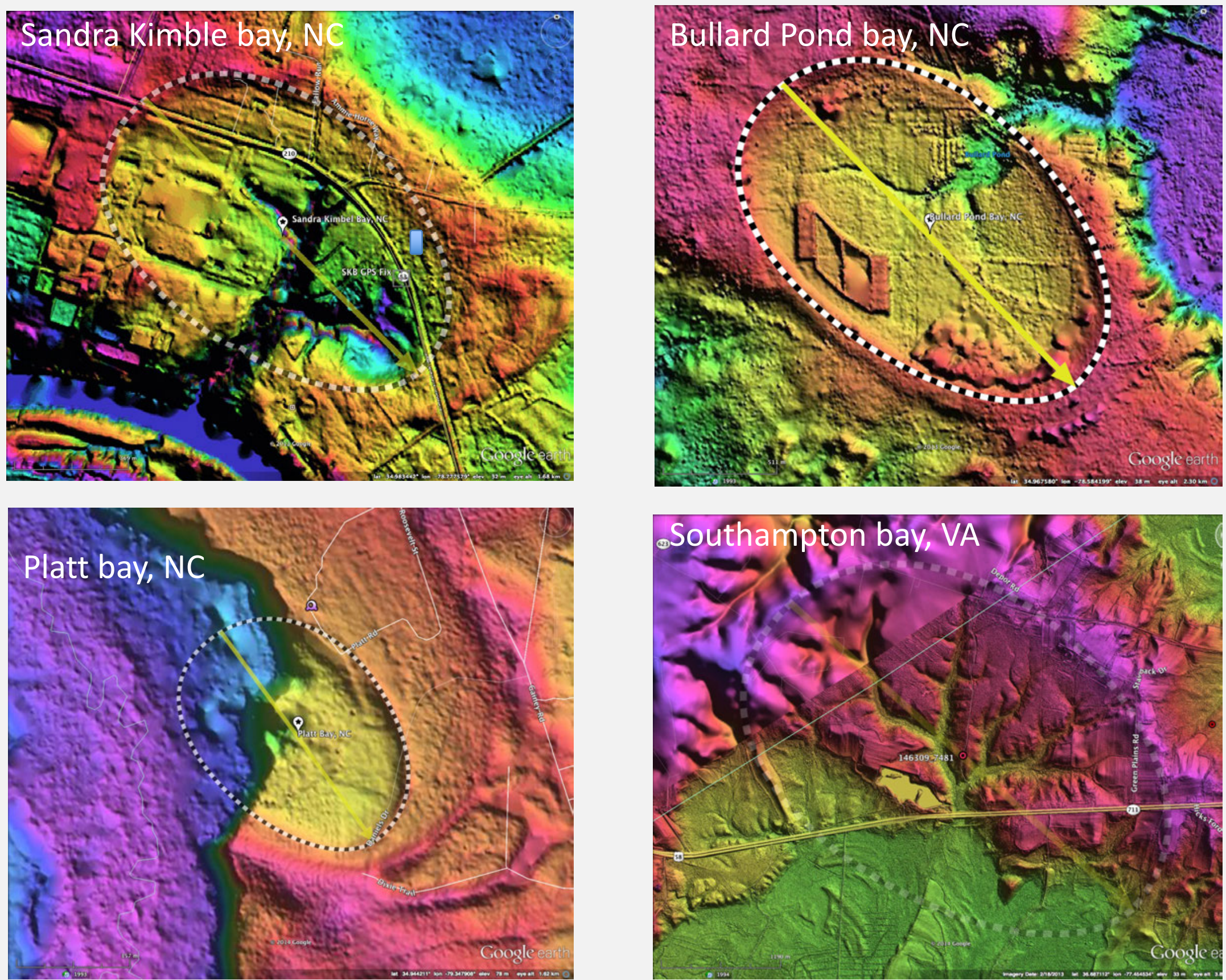


\section{Erosion Control - who's running the show?}

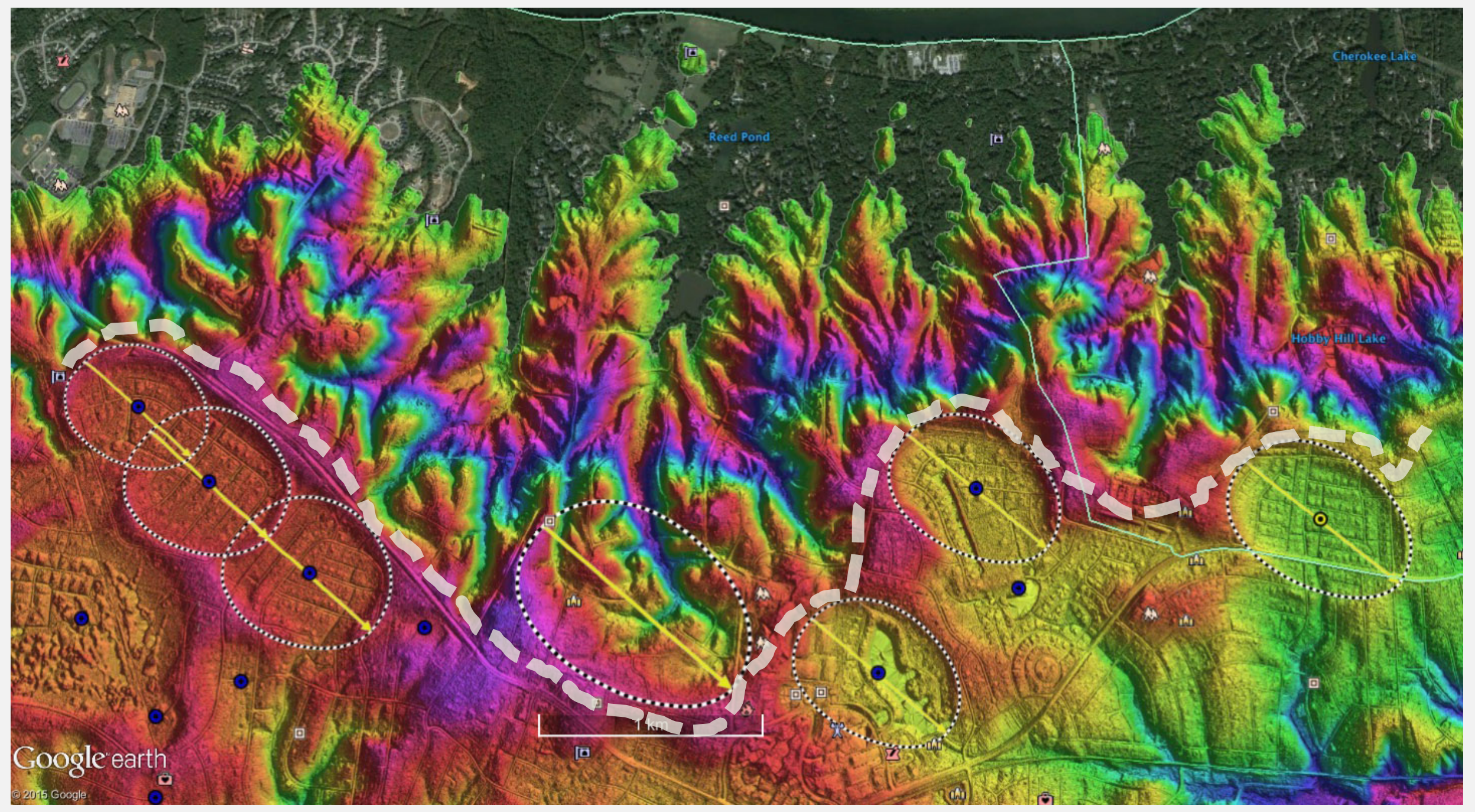

Differential erosion of the Midlothian Plateau 


\section{Bay 150310_0969, just $20 \mathrm{~km}$ West of Here}

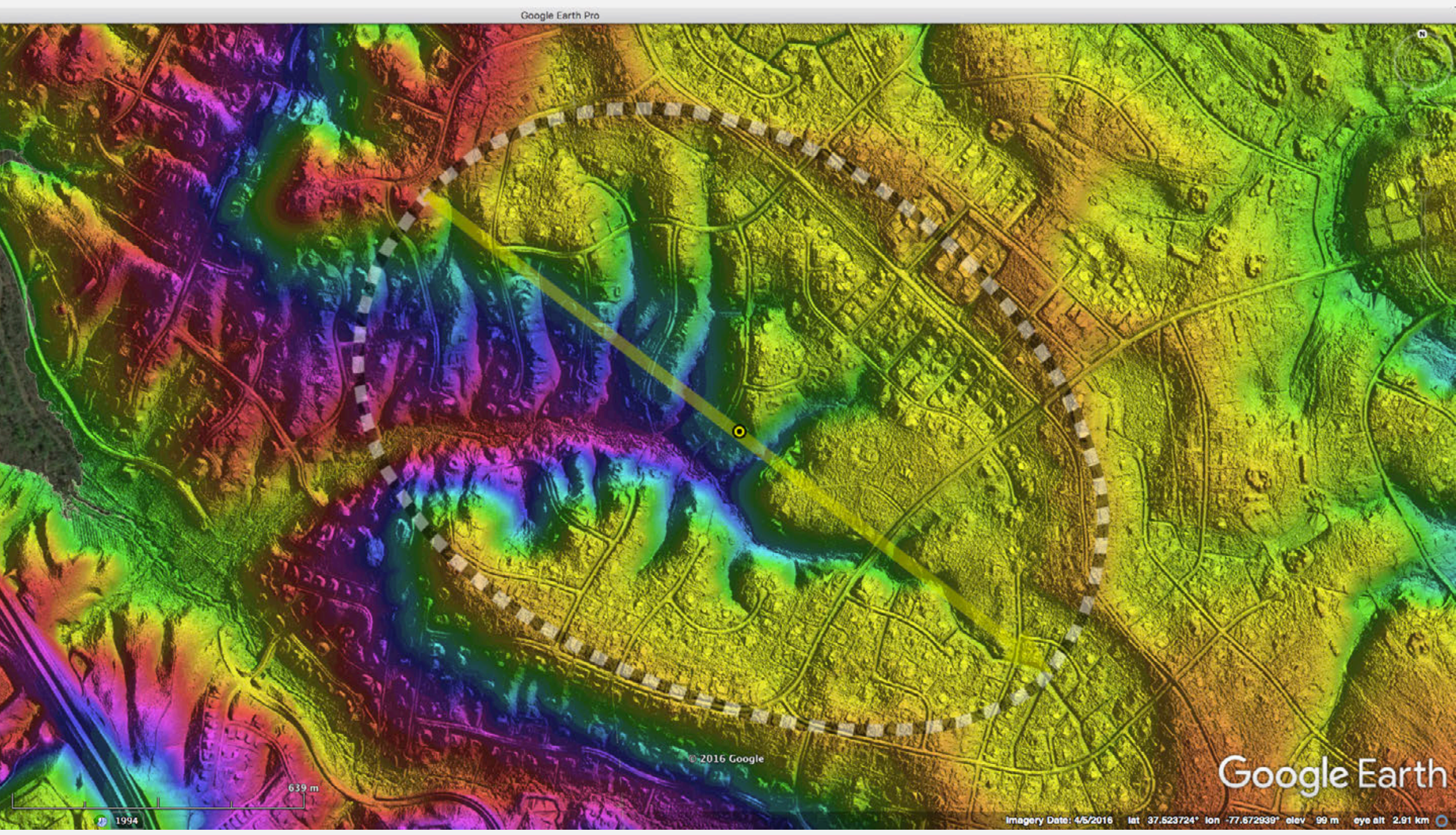

Major axis: $1.76 \mathrm{~km} \quad$ Bearing: 130을 Elevation: $96 \mathrm{~m}$ 


\section{Blythe Bay, Wilmington, NC}

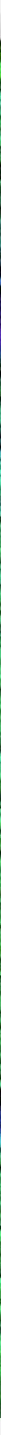

Major axis: $3.0 \mathrm{~km} \quad$ Bearing: 136을 Elevation: $13 \mathrm{~m}$ 


\section{Saginaw Impact Manifold Hypothesis}

Carolina bays are not ephemeral, wispy landforms, but rather represent the surface topology of a sheet of unconsolidated quartzose grains, deposited as ejecta during the Mid Pleistocene Transition impact event $\sim 780 \mathrm{Ka}$. The planforms and orientations have been robustly imprinted into the landscape, and have resisted ongoing erosional and accretionary processes. 


\section{Saginaw Impact Manifold Hypothesis}

Carolina bays are not ephemeral, wispy landforms, but rather represent the surface topology of a sheet of unconsolidated quartzose grains, deposited as ejecta during the Mid Pleistocene Transition impact event $\sim 780 \mathrm{Ka}$. The planforms and orientations have been robustly imprinted into the landscape, and have resisted ongoing erosional and accretionary processes.

The hypothesis seems easily falsified:

... "they don't look that old!"

... "there is no erosion!"

... "bay sediments dating does not supported a singular event!"

... "they are too far away from the MPT Impact Event!" 


\section{OSL dating fieldwork at Herndon Bay, NC}

Moore, et al documented rim construction at $36.7+/-4.1,29.6+/-3.1$, and $27.2+/-2.8 \mathrm{ka}$

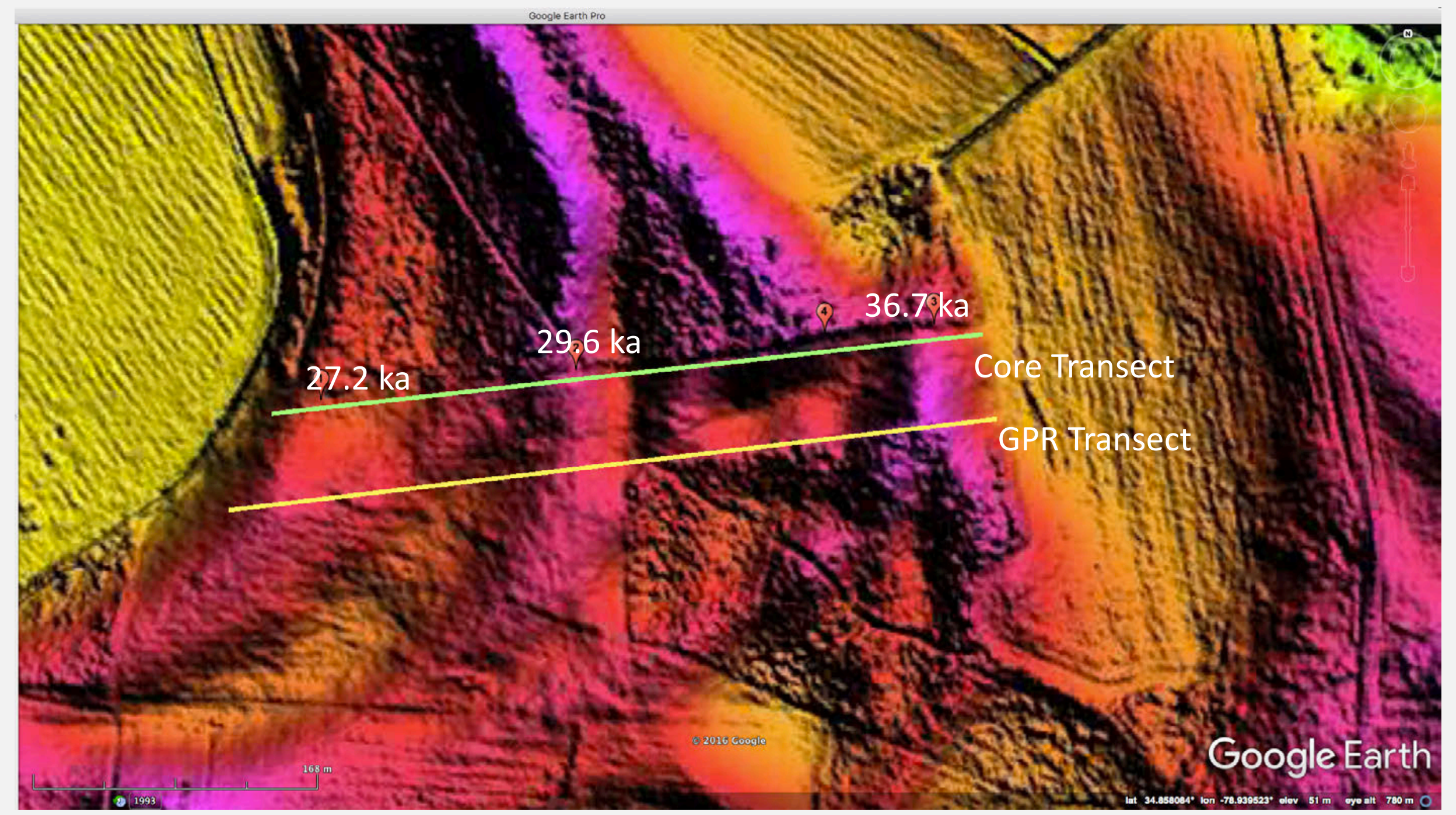

Moore, Brooks, Mallinson, Parham, Ivester And Feathers, Rapid Scour, Sand Rim Construction, And Basin Migration Of A Carolina Bay In Southeastern North Carolina, GSA Abstracts With Programs. Vol. 46, No. 3, P.96 


\section{Herndon Bay, NC}

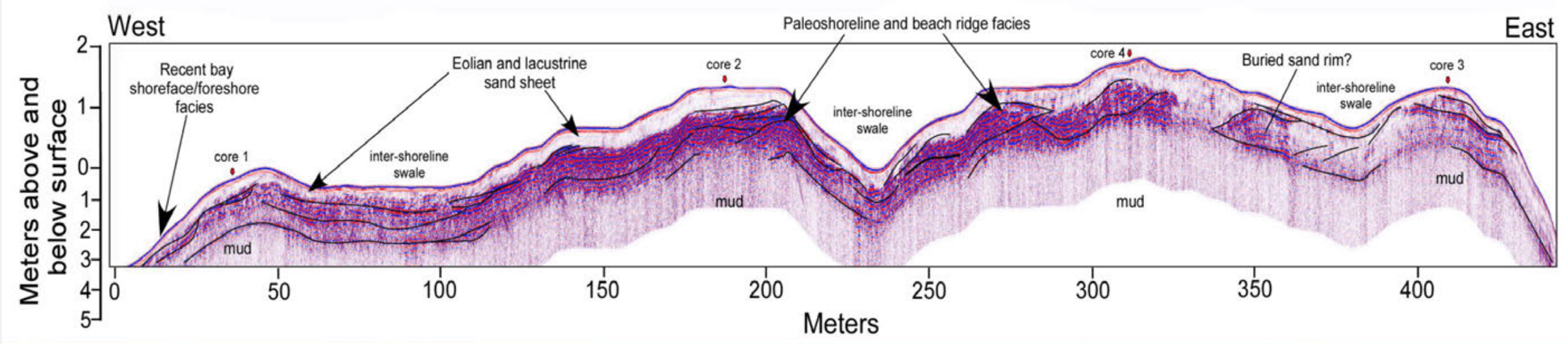

Moore's GPR trace clearly illustrates that a structure lying at depths below the sampled strata are actually controlling the relief. What's down there?

Moore, Brooks, Mallinson, Parham, Ivester And Feathers, Rapid Scour, Sand Rim Construction, And Basin

Migration Of A Carolina Bay In Southeastern North Carolina, GSA Abstracts With Programs. Vol. 46, No. 3, P.96 


\section{Herndon Bay, NC}

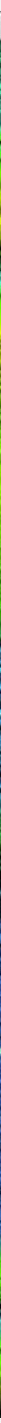

Herndon bay is on the lower right here in my LiDAR imagery. What is quite enigmatic is that it has two sibling bays which are perfect matches to the same $1.17 \mathrm{~km}$ bayCarolina overlay. Exact, just copy and place in Google Earth. 


\section{Herndon Bay, NC}

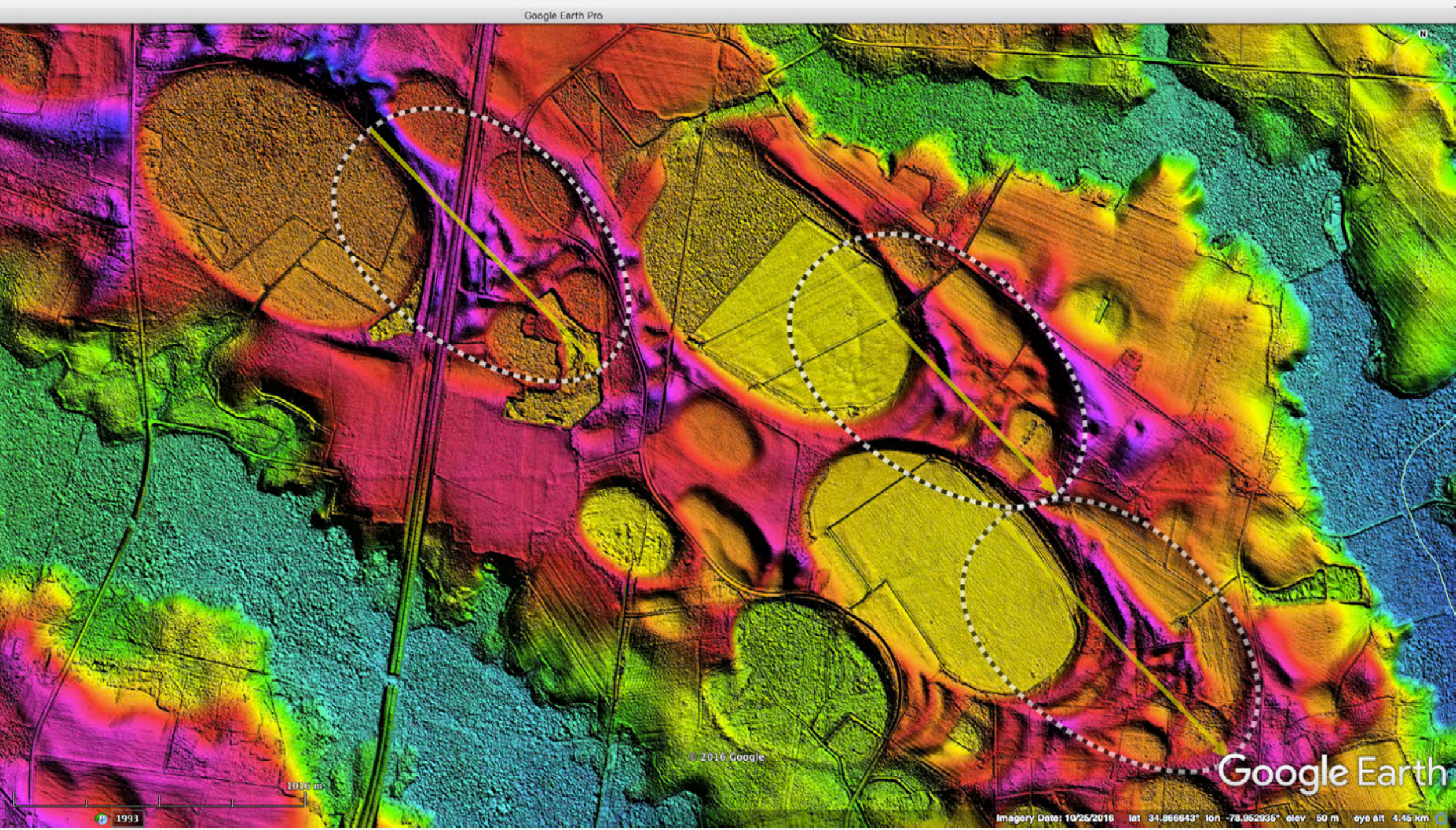

And each sibling bay has a correlated shadow bay which are perfect matches to the same 1.17 km bayCarolina overlay. Exact, just copy and place in Google Earth. 


\section{Summary}

\section{Eroded Carolina bays exhibit indications of great age}

Dissection of Costal terraces generates Valley Head Basins when

Carolina bays are penetrated by headward stream erosion

Extensive Deep Coring $(10 \mathrm{~m})$ is required to identify deposits controlling surface expression

Cosmogenic ${ }^{26} \mathrm{Al}-{ }^{10} \mathrm{Be}$ burial dating needed to reach back beyond $50 \mathrm{ka}$ to $200 \mathrm{ka}$ limits of classic dating tools 
- 2 ind

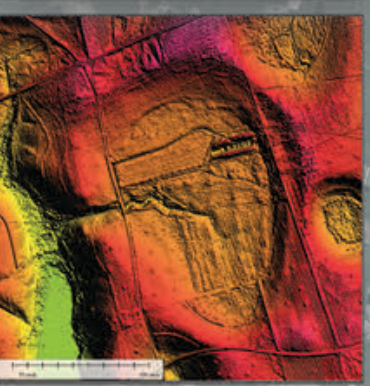

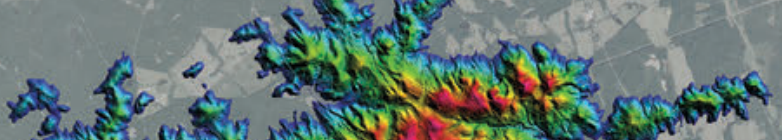

and

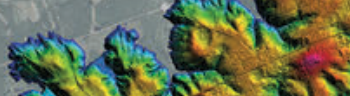

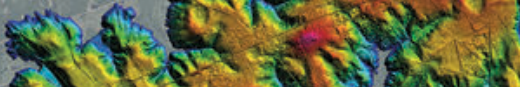

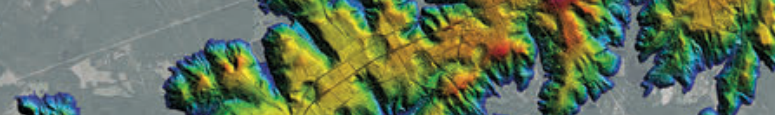
ath so war:

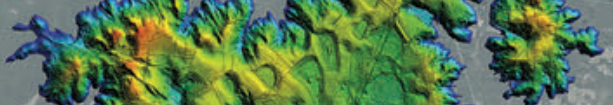
3.

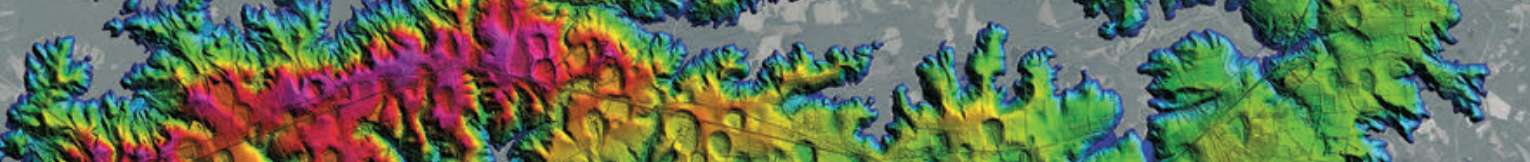

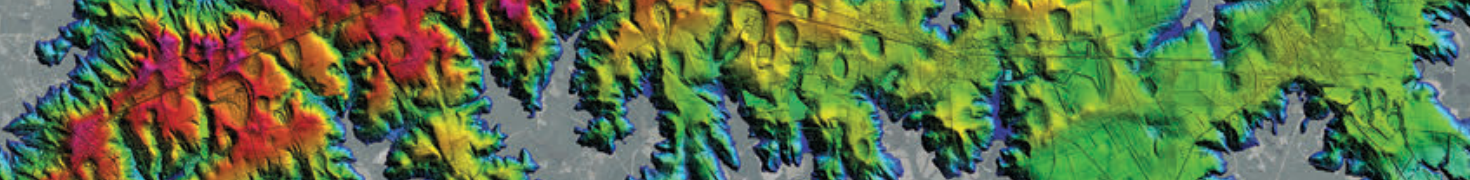

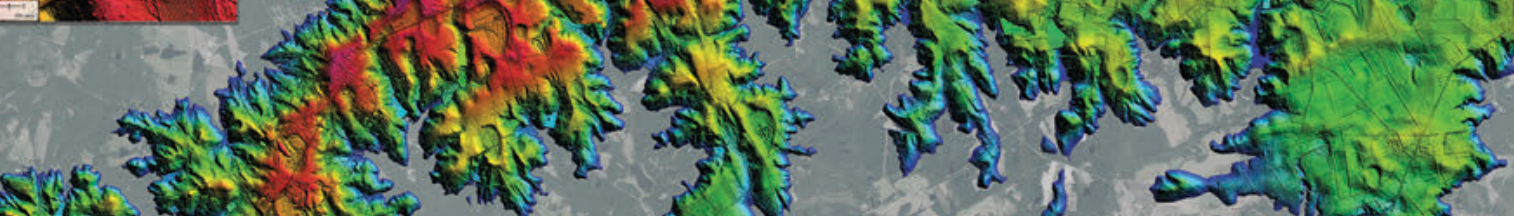
(s)

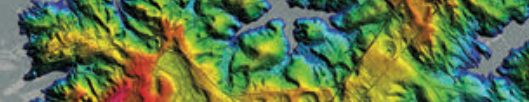
2.

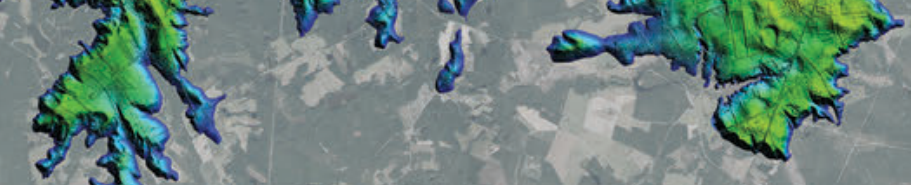
te

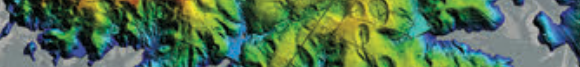

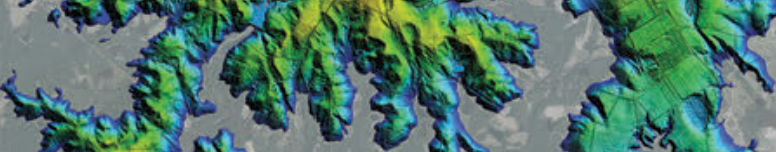
and of

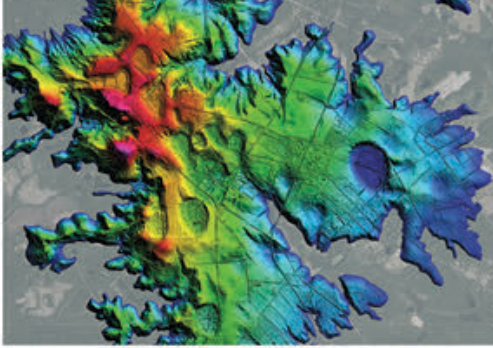
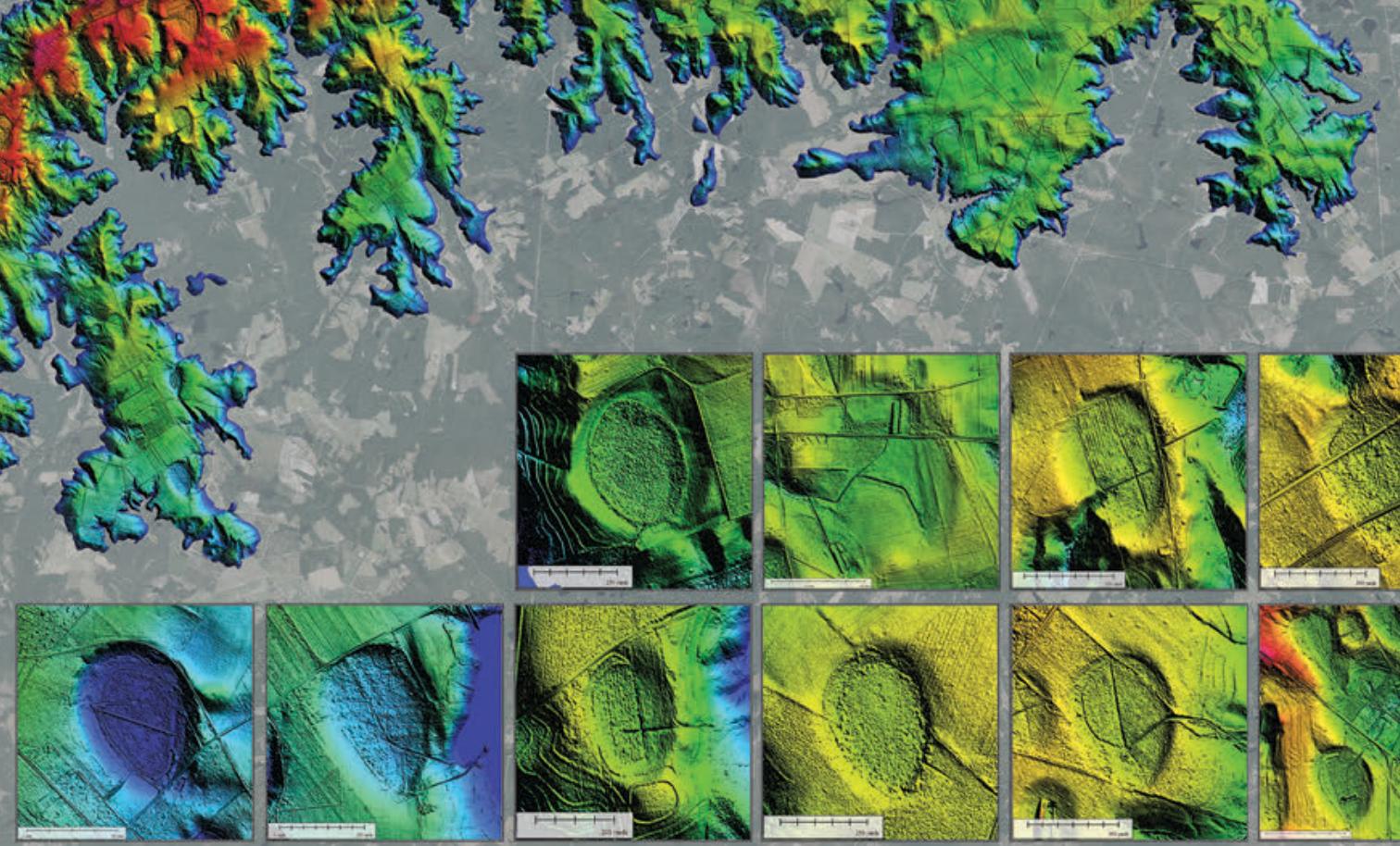

강
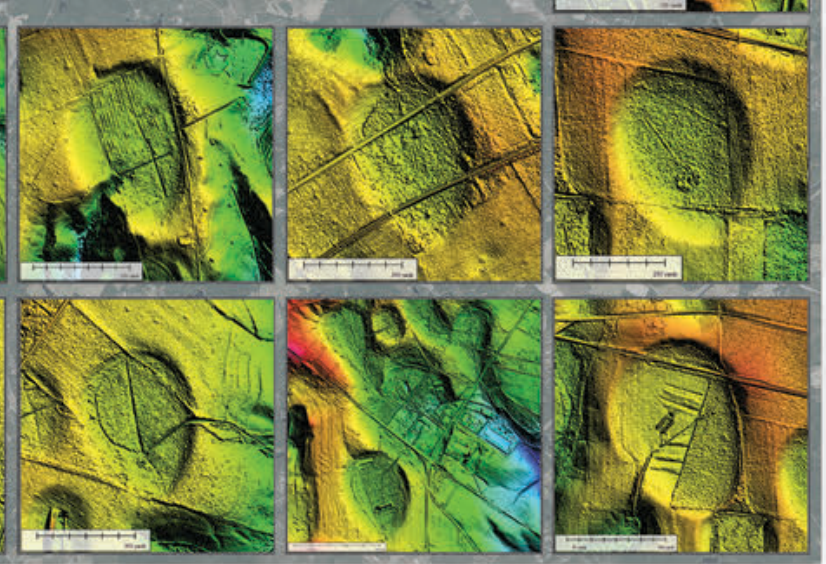

$$
\text { The Carolina Bays of Ridge Spring }
$$

